\title{
Towards an improvement in the spectral description of central stars of planetary nebulae ${ }^{\star}$
}

\author{
W. Weidmann ${ }^{1,2}$, R. Gamen ${ }^{3}$, D. Mast ${ }^{1,2}$, C. Fariña $a^{4,5}$, G. Gimeno ${ }^{6}$, E. O. Schmidt ${ }^{7}$, R. P. Ashley ${ }^{4,8}$, \\ L. Peralta de Arriba ${ }^{4,9}$, P. Sowicka ${ }^{4,10}$, and I. Ordonez-Etxeberria ${ }^{4,11}$ \\ ${ }^{1}$ Universidad Nacional de Córdoba, Observatorio Astronómico, Laprida 854, X5000BGR Córdoba, Argentina \\ e-mail: walter@oac.unc.edu.ar \\ 2 Consejo de Investigaciones Científicas y Técnicas de la República Argentina, Córdoba, Argentina \\ 3 Instituto de Astrofísica de La Plata, CONICET-UNLP, and Facultad de Ciencias Astronómicas y Geofísicas, UNLP. Paseo del \\ Bosque s/n, 1900 La Plata, Argentina \\ ${ }^{4}$ Isaac Newton Group of Telescopes, 38700 Santa Cruz de La Palma, La Palma, Spain \\ 5 Instituto de Astrofísica de Canarias (IAC) and Universidad de La Laguna, Departamento de Astrofísica, Tenerife, Spain \\ ${ }^{6}$ Gemini South c/o AURA, Casilla 603, La Serena, Chile \\ 7 Instituto de Astronomía Teórica y Experimental (IATE), Universidad Nacional de Córdoba, CONICET, Observatorio Astronómico \\ de Córdoba, Laprida 854, X5000BGR Córdoba, Argentina \\ 8 Department of Physics, University of Warwick, Gibbet Hill Road, Coventry CV4 7AL, UK \\ 9 Institute of Astronomy, University of Cambridge, Madingley Road, Cambridge CB3 OHA, UK \\ ${ }^{10}$ Nicolaus Copernicus Astronomical Center, Bartycka 18, 00-716 Warsaw, Poland \\ 11 Departamento de Física Aplicada I, Escuela de Ingeniería de Bilbao, Universidad del País Vasco, Bilbao, Spain
}

Received 20 August 2017 / Accepted 13 January 2018

\begin{abstract}
Context. There are more than 3000 known Galactic planetary nebulae, but only 492 central stars of Galactic planetary nebulae (CSPN) have known spectral types. It is vital to increase this number in order to have reliable statistics, which will lead to an increase of our understanding of these amazing objects.

Aims. We aim to contribute to the knowledge of central stars of planetary nebulae and stellar evolution.

Methods. This observational study is based on Gemini Multi-Object Spectrographs (GMOS) and with the Intermediate Dispersion Spectrograph (IDS) at the Isaac Newton Telescope spectra of 78 CSPN. The objects were selected because they did not have any previous classification, or the present classification is ambiguous. These new high quality spectra allowed us to identify the key stellar lines for determining spectral classification in the Morgan-Keenan (MK) system.

Results. We have acquired optical spectra of a large sample of CSPN. From the observed targets, 50 are classified here for the first time while for 28 the existing classifications have been improved. In seven objects we have identified a P-Cygni profile at the He I lines. Six of these CSPN are late O-type. The vast majority of the stars in the sample exhibit an absorption-type spectrum, and in one case we have found wide emission lines typical of [WR] stars. We give a complementary, and preliminary, classification criterion to obtain the sub-type of the $\mathrm{O}(\mathrm{H})$-type CSPN. Finally, we give a more realistic value of the proportion of CSPN that are rich or poor in hydrogen.
\end{abstract}

Key words. planetary nebulae: general - stars: Population II - stars: early-type - surveys

\section{Introduction}

Planetary nebulae are the end products of the evolution of stars between 0.8 and $8 M_{\odot}$, before they cool as white dwarfs (WD). After they terminate the asymptotic giant branch (AGB) phase of their evolution, the outer layers of the progenitor star are ejected and the remnant compact object begins to ionize the gaseous envelope.

Currently, there are more than 3000 planetary nebulae known in our Galaxy (Parker et al. 2012), however, spectroscopic information of the central star is available for only $16.4 \%$ of them (Weidmann \& Gamen 2011b, hereafter Paper I). Although the number of planetary nebulae candidates has been increasing quickly (Le Dû 2017; Kronberger et al. 2016; Simpson et al. 2015), spectral classification, or even the detection of

\footnotetext{
* The spectra are only available at the CDS via anonymous ftp to cdsarc.u-strasbg. fr (130.79.128.5) or via http://cdsarc u-strasbg.fr/viz-bin/qcat? J/A+A/614/A135
}

the ionizing star, has been progressing far more slowly. The most important cause of this imbalance is probably the faintness of central stars of planetary nebulae (CSPN) with respect to their nebula. These objects are intrinsically faint, with $60 \%$ of the CSPN listed in the Strasbourg-ESO Catalogue of Galactic Planetary Nebulae (SECGPN; Acker et al. 1992) having a magnitude of $V>15.5$.

In recent years, large telescopes have made it possible to observe fainter CSPN, further improving the spectral resolution. This is leading to important progress in the observational study of CSPN as it allows the identification of stellar absorption lines, especially those that are masked by the strong nebular emissions. In particular, in order to identify H-rich objects, He I and Balmer lines need to be detected, but these features are usually masked by nebular emissions. Perhaps because of the difficulty (owing to contamination from nebular emissions) in disentangling the stellar spectrum from the nebular one, only a few CSPN have been analysed using model atmosphere techniques (e.g. Frew et al. 2014). 
Central stars of planetary nebulae are hot evolved stars (prewhite dwarfs) and their optical spectra cover a range of different spectral types. Nevertheless, this group of stars is comprised of two distinct populations, so-called H-rich and H-poor (Aller 1948). Despite the similarity in their evolutionary stage, they show very different spectral characteristics. H-rich stars display Balmer and Pickering He II lines. On the other hand, the CSPN spectra of the H-poor population are dominated by the WolfRayet stars (Wolf-Rayet stars of population II are denoted by [WR]), which displays intense and wide lines of $\mathrm{N}$ III, C IV or $\mathrm{O}$ VI, depending on whether it is a [WN], [WC], or [WO].

The ratio of number of stars from both populations is not yet clear. Mendez (1991) observed that about 30\% of CSPNs are $\mathrm{H}$ deficient (with 115 objects), but the compilation presented in Paper I resulted in about $40 \%$ (with 492 objects). This difference may be due to the low number of CSPNs with reliable spectral classification considered in the mentioned studies. It is necessary to increase the observational data to obtain more robust statistics.

According to Schonberner (1989) a single star after AGB stage should retain a normal H-rich composition. However, the proportion of CSPN with hydrogen-deficient atmospheres is not negligible, thus other phenomena should be taken into account. In this sense, having a realistic ratio between these two types of CSPN will result in more robust models of stellar evolution. This is only possible by increasing the number of CSPN with spectral type determined.

In order to contribute to the understanding of this final stage in stellar evolution, we undertook this spectroscopic survey of CSPN, which is part of a series of works devoted to the spectroscopic study of CSPN. In this sense, we endeavoured to obtain high quality spectra, which would allow us to qualitatively determine the spectral classification of CSPN. In particular, to determine if their photospheres are $\mathrm{H}$-rich or $\mathrm{H}$-deficient. Section 2 describes the observations, spectrograms and reduction procedures, Sect. 3 explains the classification criteria, and Sect. 4.1 presents the spectral descriptions and classifications. We present and summarize our conclusions in Sect. 5.

\section{Observations, data reduction and sample}

A total of $78 \mathrm{CSPN}$ spectra were studied and are presented in this paper. Sixty-six CSPN were observed with Gemini Multi-Object Spectrographs (GMOS, Hook et al. 2004) at the Gemini Telescopes North and South ${ }^{1}$ (Table A.2), and twelve with the IDS spectrograph at the $2.5 \mathrm{~m}$ Isaac Newton Telescope (Table A.1). In addition, we increased the sample by adding other GMOS spectra available in the Gemini archive. All data were processed in the same way, bias frames, dome flat-fields and arc lamp exposures were taken as part of the Gemini baseline calibrations.

The GMOS observations were carried out at the $8.1 \mathrm{~m}$ Gemini South and North telescopes in their long-slit spectroscopic modes. At Gemini South, observations taken before June 2014 used the GMOS-S E2V detector array, which consists of three adjacent $2048 \times 4608$ pixel CCDs separated by two 2".8 wide gaps and a pixel scale of 0.073 arcsec pix $^{-1}$ in unbinned mode and spectral response of approximately 0.36 to $0.93 \mu \mathrm{m}$. Observations taken after June 14 used the new Hamamatsu CCDs detector array, with improved the red sensitivity (Gimeno et al. 2016). The Hamamatsu array consists of three $2048 \times 4176$ pixel

1 GMOS spectrograms were acquired under programmes GN2015A-Q-405, GS-2015AQ-98, GS-2015B-Q-103, GN-2016A-Q-97, GS-2016A-Q-74, GS-2016A-Q-101, and GS-2016B-Q-65 (PI: W. Weidmann).
CCDs separated by two gaps of 4".88 wide (61 pixels in unbinned mode) and the scale is 0.08 arcsec $\mathrm{px}^{-1}$ in unbinned mode. The spectral response is 0.36 to $1.03 \mu \mathrm{m}$. The slits used for the observations have dimensions of 330 arcsec long and 0 '.75 or $1^{\prime \prime} .5$ wide. The diffraction gratings R400 and B600 were used with the 1 '.5 slit $(R \simeq 1000)$ and the B1200 grating was used with the 0 '.75 slit $(R \simeq 2300)$. The details of the observations are shown in Table A.2. The configurations were chosen to allow us to detect some of key stellar lines from spectral classification, such as the blend of $\mathrm{C}$ and $\mathrm{N}$ ions at $4650 \AA$ (generally detectable in Of and [WR] stars), He II at 4686 and $5412 \AA, C$ III at $5696 \AA$ and $\mathrm{C}_{\text {IV }}$ at $5806 \AA$ (present in O and [WC]), key lines of hot O-type stars (4058 $\AA$ of N III, $4471 \AA$ of He I and 4089-4116 $\AA$ of Si IV) and key lines of WN (4604-4620-4944 $\AA$ of N v). The achieved signal-to-noise ratio (S/N) was up to 30 at $4600 \AA$.

The Isaac Newton Telescope (INT) observations were performed with the Intermediate Dispersion Spectrograph (IDS) at the $\mathrm{INT}^{2}$ on La Palma, Spain. IDS observations were carried out using the EEV10 and Red +2 detectors with the R632V grating (reciprocal dispersion $0.9 \AA \mathrm{px}^{-1}$ ) in a central wavelength of $5100 \AA$. This spectrograph setup provides a resolution of $R \sim 2300$ and spectral range coverage from approximately 4000 to $6200 \AA$. For each target three exposures were taken. The exposure times ranged from 1800 to $2100 \mathrm{~s}$ depending on the target's brightness. In all cases the slit was orientated along the parallactic angle.

The spectra were reduced following standard procedures, using tasks from IRAF $^{3}$ included in the GEMINI package (gemini.gmos IRAF package). The raw images were processed as follows: overscan and combined bias subtraction, flat-field correction, cosmic ray removal (IRAF task gscrrej or cosmicray), and one-dimensional spectra extraction (performing sky subtraction). The wavelength calibration was performed by fitting a four-order Legendre polynomial to the arcs. Finally, we rectified the continuum, in the science spectra, averaged the two or three spectra of each target, and shifted the combined spectra in wavelength to zero radial velocity.

The process for the subtraction of the nebular contribution to the CSPN spectra deserves a special mention here as it a delicate task. This process, indispensable for detecting possible stellar absorption lines, depends strongly on the angular size and spatial line profile of the PN. In Table A.3, we indicate if nebular subtraction was performed. In general this was possible for objects with angular size larger than 10 arcsec. The nebula spectrum was subtracted from the stellar one by interpolating the intensity of the nebular regions on opposite sides of the CSPN (normal to the dispersion). In general, we used a constant, but in more complicated cases we required a higher-order polynomial. However, in some objects this procedure did not work well (e.g. when the intensity of emission lines was not constant spatially or when the subtraction worked well for only some of the emission lines) and we decided not to subtract the nebular contribution.

For the sample presented in this paper the only selection criteria applied was to observe the brightest CSPN that had, up until these observations, lacked a spectral classification. In addition, we obtained spectra of some CSPN that in Paper I are

2 The INT is operated on the island of La Palma by the Isaac Newton Group of Telescopes in the Spanish Observatorio del Roque de los Muchachos of the Instituto de Astrofísica de Canarias.

3 IRAF is distributed by the National Optical Astronomical Observatories, operated by the Association of Universities for Research in Astronomy, Inc., under contract to the National Science Foundation of the USA. 
classified as weak emission-line star (WELS), O-type, H-rich or continuous, with the aim of improving that classification.

\section{Spectral classification}

\subsection{Main criteria}

We used the updated spectral-classification system for early $\mathrm{OB}$ stars, proposed by Sota et al. $(2011,2014)$. When possible, we determined the spectral qualifiers proposed by Sota et al. (2011, Table 3). It is appropriate to point out that the current classification system is qualitative, that is, comparing the unknown spectrum with the grid provided by Sota et al. (2011). In the case of O-type stars, we also added a label when the object was observed to be H-rich, following the notation of Mendez (1991).

Some caution is required with the identification of the $\mathrm{He}_{\mathrm{I}}$ features. A non-detection should be interpreted as either He i not being present, or present but masked by nebular emission. This leads to an uncertainty in the sub-type classification.

When we could not determine a specific spectral type, we adopted the following classification:

- $\mathrm{O}(\mathrm{H})$ : Balmer and He II absorption lines detected (but no He I nor $\mathrm{N}$ to determine sub-type).

- H-rich: Balmer lines detected but neither He I or He II.

- O: only He II absorptions lines detected (take into account that this classification includes the sub-type $\mathrm{O}(\mathrm{H}), \mathrm{O}(\mathrm{He})$ and $\mathrm{O}(\mathrm{C})$ ).

- cont.: a high $\mathrm{S} / \mathrm{N}$ spectrum in which no stellar features and He II emission lines were detected.

- Emission line: spectrum where no absorption lines are present but the observed emission lines have a stellar origin (for example C IV at $5806 \AA$ ).

\subsection{Other criteria: Lines in the yellow range}

As mentioned in Sect. 3.1, it is difficult to classify objects as $\mathrm{O}(\mathrm{H})$-type CSPN because the absorption lines are often filled with nebular emission. That is why it would be helpful to have supplementary criteria. Thus, we explored this possibility by analysing the portion of stellar spectrum that extends to wavelengths longer than $5000 \AA$, which is usually not taken into account for spectral classification in stars of population I.

In this spectral range, the CSPN classified as $\mathrm{O}(\mathrm{H})$-type show some important features that seem to be uncontaminated by nebular emissions. Between $5000 \AA$ and $6000 \AA$, some of these lines are: $\mathrm{O} v$ at $5114 \AA, \mathrm{N}_{\text {IV }}$ at 5200-5203 $\mathrm{A}, \mathrm{O}_{\text {vI }}$ at $5290 \AA$, He II at $5412 \AA, \mathrm{O}_{\text {III }}$ at $5592 \AA$ and $\mathrm{C}_{\text {IV }}$ at 5801-12 $\AA$. Of particular interest is the $\mathrm{C}$ IV line since it is, in general, of stellar origin (Górny 2014), usually wider than the nebular lines and is isolated, all factors that facilitate its identification and measurement.

With the objective of studying the possible correlation between the presence of these lines and the spectral sub-type, we selected the stars in which the spectral classification includes the sub-type (i.e. from $\mathrm{O} 3$ to B1). This sample consists of 37 objects from this study and eight taken from (Weidmann et al. 2015, hereafter Paper III). We itemize the following selected remarks:

- Ov $\lambda 5114$ : is present in absorption for objects earlier than $\mathrm{O}(\mathrm{H}) 3-5$, not detectable in $\mathrm{O}(\mathrm{H}) 6-9$, and is in emission for early B-type stars.

- Niv $\lambda$ 15200-03: these are subtle lines. We have always detected them in absorption in early O-type CSPN, O(H)3-4.

- O VI 15290: this line is always present in emission with similar intensity, so is it not useful as sub-type indicator.
- He II 25412 : is present in most spectra. Although it is possible that the nebula shows this emission, it is narrower than absorption. A surprising case is the CSPN of He 2-162, classified as B0 (see Fig. A.13), where this line seems to be in emission. We measured the equivalent width $(E W)$ of this line in the whole sample and obtained (for each range of subtypes) $E W(\mathrm{O} 3-4)=1.10 \pm 0.25, E W(\mathrm{O} 6-8)=0.84 \pm 0.24$ and $E W(\mathrm{~B} 0-1)<0.2$.

- O III $\lambda 5592$ : is present in emission in stars earlier than $\mathrm{O}(\mathrm{H}) 4$ and in absorption for stars later than $\mathrm{O}(\mathrm{H}) 7$.

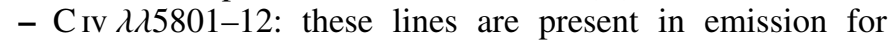
stars earlier than $\mathrm{O}(\mathrm{H}) 4$ and in absorption for stars later than $\mathrm{O}(\mathrm{H}) 7$.

We searched for the following apparent correlations among high-resolution spectra obtained within the OWN Survey (see e.g. Barbá et al. 2010, for details). We measured these lines in the spectra of the standard of classification (between O4-B0) defined by Sota et al. (2011) for Population I stars. We noted the same behaviour in the $E W$ of $\mathrm{He}$ II $\lambda 5412$; the non-detection of $\mathrm{O}$ V-VI lines; the presence of the $\mathrm{N}$ IV $\lambda \lambda 5200-03$ absorption line between the $\mathrm{O} 3$ and $\mathrm{O} 5$ spectra; the absorption line of $\mathrm{O}$ III being present in the whole sample but more intense between $\mathrm{O} 5$ and $\mathrm{O} 8.5$; the $\mathrm{C}$ IV absorption line very marginal or not detectable in the $\mathrm{O} 3$ and $\mathrm{B} 0$ sub-types, present in the other sub-types, showing a maximum in its $E W$ at O5-O7.

The $E W$ of He II $\lambda 5412$ together with the presence of the other lines of $\mathrm{O}$ III, N IV and C IV appear as promising features to discriminate, at least, between early- and late-O of population II stars, when it is not possible from the historical MK's wavelength range. The presence of the feature identified as $\mathrm{N}$ IV $\lambda \lambda 5200-03$ in population I stars was preliminarily studied by Gamen \& Niemela (2002). Further analysis is necessary to assess if useful criteria can be established using these lines.

\subsection{The qualifier " $z$ " in Pop II stars}

According to Arias et al. (2016), O-type stars that satisfy the following relations between the $E W$ of $\mathrm{He}$ II and He $\mathrm{I}$ absorption lines, $z_{4542}=E W(4686) / E W(4542) \geq 1.1$ or $z_{4471}=E W(4686) / E W(4471) \geq 1.1$, should be tagged with an additional parameter " $z$ ". It is not yet very clear what phenomenon produces the $\mathrm{O} \mathrm{Vz}$ spectral characteristic although it seems to be related with the stellar youth and low wind-strength.

We explored the determination of this qualifier in our CSPN spectra. We find that it is applicable to nine objects. All of them are earlier than O4 (A 51, AMU 1, BMP J1800-3408, HbDs 1, Lo 8, Lo 16, NeVe 3-2, PHR J1756-3342 and Sa 4-1). Moreover, we found extreme values that are even higher than those reported by Arias et al. (2016). This is the case for HbDs 1 and Lo 16 with $z_{4542}=2.2$ and $z_{4542}=2.4$ respectively. In addition, we found high $z$-values in two objects that we could not determine their sub-types - H 2-29 and Pe 1-9. In this sense, perhaps both objects deserve early-O sub-type classification.

On the other hand, we were able to identify P-Cygni profiles in seven spectra. Six of them are late O-type CSPN (H 1-65, IPHAS 190438, He 1-2, He 2-47, He 2-151 and He 2-162). The exception is the CSPN of He 2-107, despite being classified as $\mathrm{O}(\mathrm{H}) 4$, it displays P-Cygni profiles. In all these objects the P-Cygni profiles were identified in the He I lines.

According to the evolutionary sequence of H-rich CSPN, they evolve from late O-type to early O-type. In this sense, it is more likely that an object experiences mass loss in the earliest stages of its evolution, that is, late O-type. Our observational findings are in good agreement with these ideas, that is, late-O 
stars show P-Cyg profiles and as mass-loss is decreasing (and stars heat up) the spectra tend to show $\mathrm{Vz}$ characteristics, which in this case is more related to mass-loss strength.

\section{Stellar descriptions and classifications}

Spectral classifications are indicated in Table A.3 along with other parameters of interest. There, we indicate the detection, in absorption (A) or emission (E), of He II $\lambda 4542-4686-5411$, He I $\lambda 4471, \mathrm{~N} v \lambda \lambda 4603-19, \mathrm{C}$ Iv $\lambda \lambda 5801-12$, and $\mathrm{H} \beta$. We also label whether the nebula spectrum (N.S.) was subtracted. The spectra are shown in Figs. A.1-A.24, arranged according to the spectral type.

Notes on some individual CSPN spectra are included in the following section.

\subsection{Notes on individual stars}

PN G000.2-01.9 (M 2-19), Fig. A.5. Subtle Balmer absorption. Possible wide emission at $4650 \AA$ and noticeable absorption of $\mathrm{C}_{\text {III }}$ at $4647 \AA$. This is a feature present in intermediate/late O-type stars. There are absorption features of unknown origin at 4226, 4382, 4666 and $5170 \AA$ (artifact?). We classified this CSPN as $\mathrm{O}(\mathrm{H}) 6-8$. However, the lines 4226 and 4666 are observed in $\tau$ Sco, a B0-type star. In this sense, the CSPN of this object could be a $\mathrm{O}(\mathrm{H}) 8$.

PN G001.7-04.6 (H 1-56), Fig. A.22. Górny et al. (2009) report strong emission of $\mathrm{C}$ IV at $5806 \AA$, perhaps coming from stellar atmosphere.

PN G003.3-04.6 (Ap 1-12), Fig. A.13. Górny et al. (2009) classified this CSPN as [WCL]. The authors reported emission of $C_{\text {III }}$ at $5696 \AA$, we also observe this line. Nevertheless, it is not possible to be certain that the emission is stellar. We disagree with this classification, as our spectrum clearly displays absorption lines of $\mathrm{He}_{\mathrm{I}}, \mathrm{He}$ II and $\mathrm{C}$ III typical of an early B-type star. Although we could not identify the Balmer series, nebular lines are very strong. In addition, we could identify all lines reported by (Weidmann \& Gamen 2011a, hereafther Paper II). We note that this CSPN displays absorption of C IV at 5801-11 $\AA$. This feature is typical of an O late star.

$P N$ G003.5-02.4 (IC 4673), Fig. A.10. It is not easy to fit the nebular profile, however the presence of the Balmer series can be distinguished. There is He I in absorption at 4471, 4921 and $4387 \AA$. He II is not visible. Is it not clear if there is a stellar feature at $4686 \AA$. Unknown absorption at $4362 \AA$. This line is observed in $\tau$ Sco, a B0-type star. Which is compatible with our classification of the CSPN of IC 4673 as O8.

PN G005.0+03.0 (Pe 1-9), Fig. A.17. The clear lines of He II indicate that it is an $\mathrm{O}(\mathrm{H})$ star. However, according to Sect. 3.3, this CSPN could be an early O-type star.

PN G007.8-04.4 (H 1-65), Fig. A.10. This object is very strange, with a very low excitation class (EC), and no published spectra. In addition, our spectra display many emission lines. This object may not be a planetary nebula. The central star of this nebula was classified as [WC11] (Górny et al. 2009) and WELS (Acker \& Neiner 2003). However, we were able to identify key lines of a supergiant late O-type star. Emission at $4686 \AA$ has to be of stellar origin, because the EC of the nebulae is very low. Moreover, it is likely that the He II line at $4026 \AA$ displays a P-Cygni profile. This profile is visible in the He $\mathrm{I}$ lines at 4471 and $5016 \AA$. Absorption features to the left of $\mathrm{H} \varepsilon, \mathrm{H} \zeta$ and $\mathrm{H} \eta$, are probably the Balmer series of the CSPN.
PN G011.3-09.4 (H 2-48), Fig. A.18. For this object we have only detected $\mathrm{O}_{\mathrm{III}}$ in absorption at $5592 \AA$.

$P N$ G013.1+05.0 (Sa 3-96), Fig. A.21. This is a poorly studied object with a very low EC. Our two-dimensional spectra show a bright star that is not symmetrical with the nebular profile emission (therefore it is probably not the CSPN). The nebular subtraction is regular, however we can infer absorption at $\mathrm{H} \beta$. In addition, the spectrum shows absorption lines of unknown origin at 5167,5179 (N II?), and $5264 \AA$. The line at $5179 \AA$ is observed in $\tau$ Sco, a B0-type star. In this sense, the CSPN of this object could be an late O-type star.

PN G014.8-08.4 (SB 20), Fig. A.3. Clear absorption of He II at 4200 and $4542 \AA$. Moreover, $4471 \AA$ is not detected. On the other hand, the ion Ov at $5114 \AA$ is in absorption, typical of hot stars. There is absorption of unknown origin at 5125 and $5144 \AA$.

PN G016.4-01.9 (M 1-46), Fig. A.7. The spectrum displays the key lines of Si IV at $4116 \AA$ and C III at $4696 \AA$, together with O III in absorption at $5592 \AA$. These features are typical of an intermediate O-type star. In addition, the spectrum displays clear emission lines of $\mathrm{N}_{\text {III }}$ and $\mathrm{C}_{\text {III }}$ at 4634 and $4647 \AA$ respectively. Our classification agrees with that reported by Handler (2003).

PN G017.6-10.2 (A 51), Fig. A.5. There are no traces of $\mathrm{He} \mathrm{I}$, despite the high $\mathrm{S} / \mathrm{N}$. We note the clear $\mathrm{N} v$ in emission at 4603$19 \AA$. The intense absorption at $4686 \AA$ is compatible with a luminosity class V. Absorption lines are wider than the nebula, indicating an evolved object. Greenstein \& Minkowski (1964) classified this object as sd O8k. We disagree with this classification, as our spectra display clear lines of Nv, typical of an early O-type star.

$P N$ G034.3+06.2 (K 3-5), Fig. A.24. Although the object has an appreciable angular size, it is not possible to perform a good nebular subtraction. The two-dimensional spectrum shows clear emission lines in the central region of the object. Although most of them originate from the nebula, there is a fraction for which a stellar origin can not be discarded (4340, 4471, 4686, $4713,4861,5311,5412,5801-12$, and $5876 \AA$ ). This object is probably a double-envelope PN.

PN G036.4-01.9 (IPHASX J190438.6+021424), Fig. A.14. Object classified as probably PN by Sabin et al. (2014). The object is very affected by extinction (self-extinction?). There are no [O III] emissions. The star displays all Fe II lines typical of a $\mathrm{B}[\mathrm{e}]$ star.

PN G038.4-03.3 (K 4-19), Fig. A.13. The Balmer series is visible together with $\mathrm{He}$ I at 4471, 4921 and 5876 (with P-Cygni profile). In addition, the spectrum displays clear absorption of C III at $4650 \AA$ and $\mathrm{N}_{\text {III }}$ at $4641 \AA$.

PN G042.0+05.4 (K 3-14), Fig. A.9. Subtle absorption of He II at $4686 \AA$ is detected in addition to a narrow absorption of O III at $5592 \AA$. Also, the He I at $4144 \AA$ is clear.

$P N$ G044.3-05.6 (K 3-36), Fig. A.22. The spectra of this object do not show clear lines of a $\mathrm{O}(\mathrm{H})$-type star. However, present in emission are $\mathrm{O}_{\text {III }}$ at $5592 \AA$ and $\mathrm{C}_{\mathrm{IV}}$ at $\lambda \lambda 5801-12$. In addition, absorption of $\mathrm{O} v$ at $5114 \AA$ is visible. These features, according Sect. 3.2, are characteristic of a $\mathrm{O}(\mathrm{H}) 3-4$. Hence, the CSPN of K 3-36 is probably an early $\mathrm{O}(\mathrm{H})$-type star.

$P N$ G047.1+04.1 (K 3-21), Fig. A.23. The two-dimensional stellar continuum is not symmetrical with the nebular emission profile. Probable absorption of He I at 4385 and $4921 \AA$.

$P N$ G052.2+07.6 (K 4-10), Fig. A.4. This is a poorly studied object. We only detect $\mathrm{O} v \mathrm{v}$ in absorption at $5114 \AA$.

$P N$ G055.6+02.1 (He 1-2), Fig. A.9. Clear absorption of $\mathrm{He}_{\mathrm{I}}$ at 4471 and $4921 \AA, \mathrm{C}_{\text {III }}$ at $4650 \AA$, N III at 4097 and 
4511-15 $\AA$, Si IV at 4089 and $4116 \AA$ A. Finally, absorption of O III at $5592 \AA$ is evident. This is a strange feature for a late star. On the other hand, C III emission at $5696 \AA$ is evident (stellar origin?). The spectrum displays a P-Cygni profile at $5876 \AA$.

PN G060.0-04.3 (A 68), Fig. A.20. Good nebular subtraction, but poor $\mathrm{S} / \mathrm{N}$. There is a clear absorption at $\mathrm{H} \alpha$.

$P N$ G068.7+14.8 (Sp 4-1), Fig. A.22. The spectra have a very high $\mathrm{S} / \mathrm{N}$. There are absorptions of He II at $5412 \AA$ (doubtful) and $\mathrm{Ov}$ at $5114 \AA$. On the other hand, strong emission at 4650-58 $\AA, C_{\text {III }}$ at 5696, C IV 5801-12 $\AA$. The FWHM of this emission line is double that of the nebular ones. In addition, O III at $5592 \AA$ is present in emission. These features, according Sect. 3.2, are characteristic of a $\mathrm{O}(\mathrm{H}) 3-4$. Hence the CSPN of Sp 4-1 is probably an early $\mathrm{O}(\mathrm{H})$-type star.

PN G075.9+11.6 (AMU 1), Fig. A.1. Although originally classified as sdO (Østensen et al. 2010), the recent discovery of a $\mathrm{PN}$ around it (Aller et al. 2013) leads us to prefer a classification $\mathrm{O}(\mathrm{H}) 3 \mathrm{~V}$, more according to a PN central star. In addition, in our

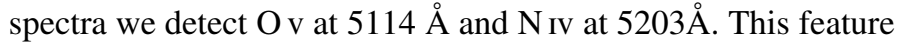
was not reported by Aller et al. (2013). Moreover, the line 4686 is very intense in relation to 4542 and 4471 is absent. In this sense we prefer a qualifier " $z$ " (Arias et al. 2016).

PN G107.6-13.3 (Vy 2-3), Fig. A.2. The lines that we detect in the optical range are listed in Table A.3. In addition, the spectra display clear absorption of $\mathrm{O} v$ at $5114 \AA$, and quite doubtful of $\mathrm{He}_{\mathrm{I}}$ at $4121 \AA$. Guerrero \& De Marco (2013) reported two lines at far-UV ( $\mathrm{S}$ VI and $\mathrm{O} \mathrm{VI}$ ), both display P-Cygni profile. These features are compatible with our classification of an early $\mathrm{O}(\mathrm{H})$-type.

PN G135.9+55.9 (TS 01), Fig. A.16. This CSPN shows a clear absorption of He II at $4686 \AA$. This feature leads us to reject a luminosity class I. We rather prefer a classification $\mathrm{O}(\mathrm{H})$ III-V. The spectrum displays clear absorption at $\mathrm{H} \gamma$ and $\mathrm{He}$ at $4471 \AA$. However, it is evident that the nebular lines are shifted with respect to the stellar ones, suggesting a binary core. Multiplicity of this CSPN was reported by Napiwotzki et al. (2005). On the other hand, Tovmassian et al. (2004) by means of UV spectra, classified this object as WD/NS. Nevertheless, we cannot give an accurate description of the spectral type and we see no evidence of wide absorption lines.

PN G184.0-02.1 (M 1-5), Fig. A.24. This is a young PN. It is difficult to infer some features of the CSPN. We detect narrow emission lines only at 4686 and $5806 \AA$. Although we can not be sure that these features come from the star.

PN G199.4+14.3 (PM 1-29), Fig. A.12. There is a possibility that it is a proto-planetary nebula. This object was studied by Arkhipova et al. (2006), who classified the CSPN as an early B-type.

PN G211.2-03.5 (M 1-6), Fig. A.8. This CSPN was classified as a possible [WC10-11] (see, Paper II). However, we can now identify He II absorption lines. The nebula is of low EC, so the nebular feature at $4686 \AA$ has to be very weak or absent. In our spectra the line 4686 is weak, thus the stellar feature of He II must be weak too. In this sense, we classified this CSPN as late OI. In addition, absorption of O III at $5592 \AA$ is detected.

PN G234.9-01.4 (M 1-14), Fig. A.15. This CSPN was classified as O-type in Paper II. Now, with a better quality spectrum, we could detect the Balmer lines. In addition we detect absorption of $\mathrm{O}_{\text {III }}$ at $5592 \AA$ and $\mathrm{C}_{\text {IV }}$ at 5801-12 $\AA$. Both features are typical of a late $\mathrm{O}$. Also the spectrum displays emission of $\mathrm{C}$ III at $5696 \AA$ (stellar origin?).
PN G238.0+34.8 (A 33), Fig. A.19. This object has wide absorption lines (FWHM of $\sim 20 \AA$ ). He II at $4200 \AA$ is not detected. It is expected to be an evolved star, the ionizing object of an old PN as A 33. Aller (1975) classified this CSPN as sd Op and Mendez (1991) reported a spectral type $\mathrm{O}(\mathrm{H})$. Our spectrum, of better quality, allows us to determine that the FWHM of the absorption lines are comparable to WD stars. Our classification is similar to that obtained by Aller (1975). We confirm detection of $\mathrm{H}$ reported by Mendez (1991).

PN G249.8+07.1 (PHR J0834-2819), Fig. A.19. The slit intersected two stars lying at the geometrical centre of the PN. We confirmed that the CSPN is the brighter of the two. The other star is of a late type. The spectrum has a low $\mathrm{S} / \mathrm{N}$. Although the lines appear to be broad, it seems to be an evolved object. This is consistent with the nebula's large angular size.

PN G258.5-01.3 (RCW 24), Fig. A.19. Our spectrum displays broad lines of the Balmer series. Frew et al. (2006) report an uncertain absorption feature near $4650 \AA$ that we do not detect, despite the high $\mathrm{S} / \mathrm{N}$ of our spectrum.

$P N$ G273.6+06.1 (HbDs 1), Fig. A.1. The CSPN of this nebula is a clear $\mathrm{O}(\mathrm{H}) 3$. Moreover, we detect emission lines of $\mathrm{N} \mathrm{v}$ at 4603-19 $\AA$ (in general this line appears in absorption), $C_{\text {IV }}$ at 4658, 5801-11 , and strong O vi at $5290 \AA$. All these indicate that this is an extremely hot star. Moreover, the line 4686 is very intense in relation to 4542 and 4471 is absent. In this sense we prefer a qualifier " $z$ " (Arias et al. 2016).

PN G281.0-05.6 (IC 2501), Fig. A.8. Guerrero \& De Marco (2013) report three lines at far-UV (S vi, O vi and P v), all display P-Cygni profile. These features are compatible with an early O-type star. In addition, we observe that the nebular emissions are shifted with respect to the stellar features (binary?).

PN G285.6-02.7 (He 2-47), Fig. A.10. The spectrum has a high $\mathrm{S} / \mathrm{N}$. There is absorption of $\mathrm{O}_{\text {III }}$ at $5592 \AA$. The feature of $\mathrm{He}_{\mathrm{I}}$ at 4471, 4713, 4921 and $5876 \AA$, presents a P-Cygni profile. Absorption of the Balmer series can be inferred. It is possible that $\mathrm{He}$ II at $4026 \AA$ presents a P-Cygni profile. In addition, there are strong emission of $\mathrm{C}_{\text {II }}, \mathrm{C}_{\text {IIII, }}$ and $\mathrm{C}_{\text {IV }}$ near $4650 \AA$ that could be of stellar origin. In Paper II we classified this CSPN as [WC10-11], however our new high quality spectrum displays key absorption lines of a late O-type star. It is not possible to be sure that the emission of $\mathrm{C}_{\text {III }}$ at $5696 \AA$ is stellar.

PN G285.7-14.9 (IC 2448), Fig. A.4. Nebular subtraction is not good, for example in the strongest lines at [O III]. Nevertheless, the absorption of $\mathrm{Nv}$ at 4604-20 $\AA$ and the emission of C IV at 5801-12 $\AA$ are clear features typical of an early O-type star.

PN G288.7+08.1 (ESO 216-2), Fig. A.5. This is a poorly studied object. We classified the CSPN of this ring nebula as early O-type star. The absorption at $4686 \AA$ rejects a luminosity class I, and absence of $\mathrm{N} v$ at 4603-19 $\AA$ suggests an object later than $\mathrm{O}(\mathrm{H}) 4$. Moreover, emission of O vi at $5290 \AA$ indicates a hot star.

PN G302.2-03.1 (PHR J1244-6601), Fig. A.20. The bright central star probably does not correspond to the CSPN. The S/N of the spectrum is high. Balmer lines are narrow, suggesting an evolved star.

$P N$ G310.3+24.7 (Lo 8), Fig. A.2. Spectra display emission of C IV at $4658 \AA$ and O VI at $5290 \AA$, together with absorption of $\mathrm{O} v$ at $5114 \AA$. These are features of a very hot star. In addition, there are some absorption features of unknown origin at 4441, 5572 (O v?), 5581 (O v?), and 5598 (O v?). 
PN G312.6-01.8 (He 2-107), Fig. A.4. Strong emission of $\mathrm{C}_{\text {II, }} \mathrm{C}_{\text {III, }}$ and $\mathrm{C}_{\text {IV }}$ around $4650 \AA$, that could be of stellar origin. The nebular EC is low, so there should be no nebular emission at $4686 \AA$. Thus this feature have to be stellar, in this sense we assign to this star a luminosity class I. Significant P-Cygni profile at $\mathrm{He}_{\mathrm{I}}$ lines. Besides lines reported in Table A.3 the spectrum displays an absorption of N IV at $5203 \AA$. This line is present in early O-type stars of population I (Gamen \& Niemela 2002). This object was classified as [WC10-11] in Paper II. Nevertheless the present high quality spectrum leads us to reject such a classification.

PN G315.4-08.4 (PHR J1510-6754), Fig. A.16. The CSPN of this true planetary nebulae has been identified by Parker et al. (2006). It has a large angular size and low surface brightness In this case we hoped to find an evolved CSPN, such as a WD. However, the spectrum of this CSPN is dominated by narrow emission lines, especially $\mathrm{N}_{\text {III }}$ and $\mathrm{C}_{\text {III }}$, and an absorption feature is seen at $\mathrm{H} \beta$. The very narrow absorption, which may be covered by the emission, suggests a low surface gravity. It is surprising that having strong emission lines, these are so narrow. Perhaps this star is binary and we see the emission arising from another part of the system, such as is seen in cataclysmic variables. Nevertheless, the All-Sky Automated Survey for SuperNovae $^{4}$ (ASAS-SN, Shappee et al. 2014) does not show photometric variation.

A search of the literature revealed two cool [WC] that display similar spectra. Goodrich \& Dahari (1985) and Hu \& Bibo (1990). However, in these cases both objects are compact, so it is difficult to be sure if the lines are nebular or stellar. Another object that displays a similar spectrum is HD120678, a hot star of population I. Undoubtedly, this object requires a more detailed study.

PN G315.7+05.5 (LoTr 8), Fig. A.24. Wide emission of C IV and $\mathrm{He}_{\mathrm{II}}$ at 4658 and $4686 \AA$ respectively. Clear features of a [WR] star, which is consistent with the high EC of the nebula.

PN G317.2+08.6 (PHR J1424-5138), Fig. A.2. This is a poorly studied object. The spectra display clear features of an early $\mathrm{O}(\mathrm{H})$-type star. The strong emission of $\mathrm{N}$ IV at $4058 \AA$ is more compatible with a luminosity class I. In addition, the spectrum displays an intense absorption of N IV at $5203 \AA$.

$P N$ G323.9+02.4 (He 2-123), Fig. A.18. The spectrum of this CSPN is of low $\mathrm{S} / \mathrm{N}$. The clearest feature is He II at $4542 \AA$. In addition, $\mathrm{N} \mathrm{v}$ at $4603-19 \AA$ is detectable, this feature is typical of early O-type stars. Nevertheless, the line at $4603 \AA$ shows a profile of emission plus absorption.

PN G323.6-04.5 (WKK136-337), Fig. A.3. Strong absorption of $\mathrm{O} v$ at $5114 \AA$, a feature typical of hot objects. In addition, emission of Si IV and C IV at 4089 and $4658 \AA$ respectively. According to Sota et al. (2011), emission at $4658 \AA$ is not present in O-type stars of population I. In addition the spectra display absorption of unknown origin at 4120-25 $\AA$ (O v?).

$P N$ G325.8-12.8 (He 2-182), Fig. A.11. This is an intermediate $\mathrm{O}(\mathrm{H})$-type star. It is possible to infer an stellar absorption at $\mathrm{H} \delta$. In addition, clear absorption of $\mathrm{O}_{\text {III }}$ at $5592 \AA$ is detected.

PN G326.0-06.5 (He 2-151), Fig. A.14. In this object's spectra, the absorption at $\mathrm{H} \epsilon$ is clear. In addition, absorption of $\mathrm{O}_{\text {III }}$ at $5592 \AA$ is detected. P-Cygni profiles are detected at 5016 and $5876 \AA$.

$P N$ G326.4+07.0 (NeVe 3-2), Fig. A.6. This is a poorly studied object. The spectrum of this CSPN displays clear features of

\footnotetext{
4 http://www . astronomy.ohio-state.edu/ assassin/
}

an early O-type star. In addition, a strong absorption of $\mathrm{N}$ IV at $5203 \AA$ is detectable.

PN G329.0+01.9 (Sp 1), Fig. A.6. The subtraction of the nebular component is very good. There is emission of $\mathrm{O}$ VI at $5290 \AA$ and possible absorption of O v at $5114 \AA$. These are typical of early O-type stars, which is consistent with the absence of He I. Emission lines of N III at 4634-40-42 $\AA$ and C III at 4647-50-51 A, lead us to prefer an "(fc)" qualifier. However, the

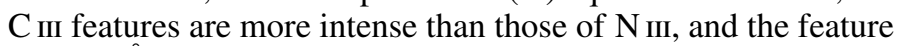
at $4686 \AA$, with emission plus absorption. This is very strange. Mendez et al. (1988) classified this object as $\mathrm{O}(\mathrm{H})$, and reported emission lines in the region around $4650 \AA$. Particularly interesting is the emission of Si IV at $4630-55 \AA$ that we detect too. It is in good agreement with the classification that we propose.

The apparent asymmetric shape in $\mathrm{H} \beta$ and $\mathrm{H} \gamma$ absorptions (blue smooth wing and narrow red), is caused by the He II absorptions at 4859 and $4338 \AA$ respectively. This feature is reported by Mendez et al. (1988).

$P N$ G329.5+01.7 (VBRC 7), Fig. A.21. It is a poorly studied object. Our nebular subtraction is not good, however, the Balmer absorption seems real. We cannot detect any other stellar features.

PN G331.4-03.5 (He 2-162), Fig. A.13. Absorption lines that display the spectrum are compatible with a B0 star. Moreover, this classification agrees with the low $T_{\text {eff }}$ reported by Mendez et al. (1992). In addition, P-Cygni profile is detect at $4650 \AA$.

PN G338.1-08.3 (NGC 6326), Fig. A.11. We performed a nebular subtraction, although with not very good results. This makes the $4471 \AA$ and $5806 \AA$, features uncertain. We infer that emissions around $4650 \AA$ are of stellar origin.

PN G349.3-04.2 (Lo 16), Fig. A.3. We classified this CSPN as early $\mathrm{O}(\mathrm{H})$-type. In addition we detect emission of $\mathrm{O}$ VI at $5290 \AA$, which is consistent with a hot star. Moreover, the stellar emission of $\mathrm{C}_{\text {III }}$ and $\mathrm{N}_{\text {III }}$ at $4650 \AA$ suggests an (fc) qualifier. The line at $4542 \AA$ is clearly wider than the other He II lines.

$P N$ G353.7+06.3 (M 2-7), Fig. A.18. The spectrum only displays a couple of absorption lines of He II. In addition, a wide absorption line of unknown origin at $5176 \AA$ can be detected.

PN G354.5-03.9 (SAB 41), Fig. A.21. The only stellar feature that we can detect of this CSPN is a strong and wide absorption ( $32 \AA$ ) at $\mathrm{H} \beta$. According to Miszalski et al. (2009a) the nucleus of this planetary nebula is likely a binary system.

PN G357.0-04.4 (PHR J1756-3342), Fig. A.3. This weak object was classified as likely planetary nebulae according to the MASH (Miszalski et al. 2008) catalogue. The central star of this object is clearly an early $\mathrm{O}(\mathrm{H})$-type star, nevertheless, its spectra displays wide lines of $\mathrm{He}$ II and $\mathrm{H}$. In particular, the line 4542 is wider than 4686.

PN G357.1-05.3 (BMP J1800-3408), Fig. A.6. We do not detect nebular emission. We note clear absorption of $\mathrm{N}$ IV at $5203 \AA$ and subtle absorption of $\mathrm{C}_{\mathrm{IV}}$ at $4658 \AA$. Both are features of a hot O-type star. Moreover, I(4686) $>\mathrm{I}(4542)$ so the luminosity class is $\mathrm{V}$.

PN G357.6-03.3 (H 2-29), Fig. A.15. Nebular emission masks the $\mathrm{H}$ lines, however, the absorption at $\mathrm{H} \delta$ is clear. There is an absorption of unknown origin at $4382 \AA$. On the other hand, according to the described in Sect. 3.3, this CSPN could be an early O-type star.

PN G358.7-03.0 (K 6-34), Fig. A.7. The spectrum displays emission of Si IV at $4116 \AA$, that suggests an early $\mathrm{O}(\mathrm{H})$-type star. The absence of $\mathrm{N} v$ rejects an $\mathrm{O}(\mathrm{H}) 3$ star. 


\subsection{Discussion}

We were able to determine sub-types for the first time for many CSPN. Sometimes it was possible to apply some non-standard criteria which should be discussed in future works. For example, ten objects from our sample were previously classified by Mendez (1991) as O(H) CSPN. These objects were included in our sample with the goal of improve such classification by adding a sub-type. In all cases (A 33, A 51, HbDs 1, He 2-151, He 2-162, He 2-182, IC 2448, Lo 8, Sa 4-1, and Sp 1) our spectra confirm the presence of $\mathrm{H}$ and He II. In this sense, we agree with classification reported by Mendez (1991).

As many times the lines used as criteria for the classification of Pop I stars are contaminated by nebular lines, it might be useful to analyse the behaviour of other stellar lines. To that purpose, we analysed some identified absorption lines (see Sect. 3.2).

Some lines, noted in a few spectra, remained unidentified. These features are, in general, of low S/N, and in the two dimensional images are not clearly detectable. Nevertheless, for those objects in which we did not subtract the nebular contribution, we are sure that the line is real and not a residual of the subtraction. Also, they were observed in more than just one spectra, which reinforces the fact that these features are not artifacts. We discarded them as diffuse interstellar bands and telluric features as we did not find them in the works of Herbig (1995), nor as telluric features in Hanuschik (2003). In order to identify these features with known ions, we used Moore (1945) and the NIST Atomic Spectra Database (Kramida et al. 2017). No transitions correspond to ions of the $\mathrm{C}$ IV, $\mathrm{O} v$, or $\mathrm{O}$ vi that are typical ions of hot stars.

It is widely known that the spectra of CSPN and Population I stars are very similar, specially the WR and [WR] stars which are indistinguishable. Perhaps the object M 1-67 is a good example that shows the similarities between these two groups of stars (Crawford \& Barlow 1991), and we also recall the case of HD 151853 which was considered in the literature as either a post-AGB or a massive star (Gamen et al. 2015). As far as we know, there are few studies analysing whether O-type stars of population I and II display different spectrum. The reason for this could be in the lack of population II O-type stars with precise spectral classification performed, that is, sub-types determined. Our current improved database of CSPN spectra allows us to perform some analysis in this direction.

A necessary warning. There are evidence that some CSPN of our sample are close binaries, most which have irradiated secondaries. Emission lines that come from the system might influence our classifications. These objects are: Pe 1-9, M 2-19, H 2-29, K 6-34 and BMP J1800-3407 (Miszalski et al. 2009b); NGC 6326 (Miszalski et al. 2011); Sp 1 (Hillwig et al. 2016); Lo 16 (Hillwig etal. 2017); PHR J1510-6754 (Hillwig etal. 2013).

\section{Summary and conclusions}

We have obtained and analysed high quality optical spectra using GEMINI and INT telescopes. We performed a qualitative determination of the spectral types of $78 \mathrm{CSPN}$, which represents an increase of $18 \%$ of the objects catalogued in Paper I. The quality of these spectra was essential in order to be able to provide subtypes to the spectral classification. We were not able to do this in our previous work - Paper II.

Fifty CSPN spectra were classified for the first time. Five objects previously classified as WELS (H 1-56, He 2-123, M 1-46, NGC 6326 and Sp 4-1) were re-classified as O-type stars, reaffirming the results obtained in Paper III. We have detected several CSPN with uncommon spectral types. For example, IPHASX J190438.6+021424 and PHR J1510-6754 were classified as $\mathrm{B}[\mathrm{e}]$ and $\mathrm{O}(\mathrm{H}) \mathrm{e}$, respectively. These objects require more detailed observations since they might be transitional objects. Ap 1-12, H 1-65, He 2-47, He 2-107, and M 1-6, previously classified as H-poor, were re-classified as H-rich. K 3-66, previously classified as continuous, was reclassified as O-type star. This fact reinforces the hypothesis of Kudritzki et al. (1981) that these objects are expected to be $\mathrm{H}$-rich. The classification of another twelve CSPN, previously classified as just $\mathrm{O}(\mathrm{H})$, were improved by adding a sub-type and, in some cases, their luminosity class. Finally, in seven objects we identified P-Cygni profile at He I lines. Six of these CSPN are late O-type. On the other hand, in nine early O-type CSPN we found evidence that they are not undergoing mass loss. According to the evolution sequence of H-rich CSPN, these evolve from late O-type to early O-type. In this sense, it is more likely that an object experiences mass loss in the earliest stages of its evolution, that is, late O-type. This is in complete agreement with that we have found.

It is surprising that, in this large spectral sample of CSPN, only one object was classified as [WR], especially since our selection criteria for the sample was based, mainly, on the apparent brightness of the star. This may be due to a selection effect.

The spectral range of our data, which includes 5000-6000 A, allowed us to study the behaviour of several lines of stellar origin, and explored they as indicators of sub-types of the $\mathrm{OB}(\mathrm{H})$-type CSPN. The importance of these lines is that they are easily identifiable (they are not contaminated by nebular features), their origin is rarely nebular, and they fall in the spectral range in which modern CCDs are most sensitive. The results are promising but further observations and analysis are needed to define more robust classification criterion with these lines.

The catalogue of Paper I lists $20 \mathrm{O}(\mathrm{H})$-type CSPN with subtype determined. With this work plus the object of Paper III, we have added 45 CSPN to the list. This improved sample will allow population analysis to be carried out. For example, contrasting the observed distribution of sub-types with those predicted from a theoretical sample of low- and intermediate- mass stars evolving as recently proposed by Miller Bertolami (2016).

Finally, with respect to the ratio $\mathrm{H}$-rich/H-poor CSPN, the simplest evolutionary models predict that there should be no $\mathrm{H}$ poor CSPN because the star leaves the AGB before it runs out the photospheric hydrogen. Then other explanations are required: born-again or evolution in an interacting binary system. In this sense, empirical information on the percentage of $\mathrm{H}$-poor is basic data to verify whether the predictions of the most complex models are fulfilled. These present new spectral classifications, together with those of Paper III, helps us to determine with better precision, the ratio between $\mathrm{H}$-rich and H-poor CSPN. In Paper I we reported H-rich/H-poor $=1.44$ (reject the wels stars). Adding these new classifications to the catalogue of Paper I we found that the ratio $\mathrm{H}$-rich/H-poor $=2.07$. Clearly, our current ratio is different to the one obtained in Paper I.

We hope that the spectroscopic data presented here will be useful as a guide for future observations that help us to understand better the final stages of stellar evolution for ordinary mass stars.

Acknowledgements. We would like to thank our anonymous referee whose critical remarks helped us to substantially improve this paper. Part of this research was supported by grant PIP 112-201201-00298 (CONICET), and by grant SeCyT UNC project NRO PIP: 30820150100067CB. Based on observations obtained 
at the Gemini Observatory, which is operated by the Association of Universities for Research in Astronomy, Inc., under a cooperative agreement with the NSF on behalf of the Gemini partnership: the National Science Foundation (United States), the National Research Council (Canada), CONICY' (Chile), Ministerio de Ciencia, Tecnología e Innovación Productiva (Argentina), and Ministério da Ciência, Tecnologia e Inovação (Brazil). RPA acknowledges funding from the European Research Council under the European Union's Seventh Framework Programme (FP/2007-2013)/ERC Grant Agreement no. 320964 (WDTracer). This research has made use of SAO Image DS9, developed by Smithsonian Astrophysical Observatory. This research made use of the SIMBAD database, operated at the CDS, Strasbourg, France. W.W. would like to thank Roberto Méndez.

\section{References}

Acker, A., \& Neiner, C. 2003, A\&A, 403, 659

Acker, A., Marcout, J., Ochsenbein, F., et al. 1992, The Strasbourg-ESO Catalogue of Galactic Planetary Nebulae. Parts I, II. (Garching: European Southern Observatory)

Aller, L. H. 1948, ApJ, 108, 462

Aller, L. H. 1975, Mem. Soc. Roy. Sci. Liège, 9, 271

Aller, A., Miranda, L. F., Ulla, A., et al. 2013, A\&A, 552, A25

Arias, J. I., Walborn, N. R., Simón Díaz, S., et al. 2016, AJ, 152, 31

Arkhipova, V. P., Klochkova, V. G., Chentsov, E. L., et al. 2006, Astron. Lett. 32,661

Barbá, R. H., Gamen, R., Arias, J. I., et al. 2010, in Rev. Mex. Astron. Astrofís. Conf. Ser., eds. T. Rivinius, \& M. Curé, 38, 30

Crawford, I. A., \& Barlow, M. J. 1991, A\&A, 249, 518

Frew, D. J., Parker, Q. A., \& Russeil, D. 2006, MNRAS, 372, 1081

Frew, D. J., Bojičić, I. S., Parker, Q. A., et al. 2014, MNRAS, 440, 1345

Gamen, R., \& Niemela, V. 2002, in Rev. Mex. Astron. Astrofís. Conf. Ser., eds. J. J. Claria, D. Garcia Lambas, \& H. Levato, 14, 16

Gamen, R., Putkuri, C., Morrell, N. I., et al. 2015, A\&A, 584, A7

Gimeno, G., Roth, K., Chiboucas, K., et al. 2016, in Ground-based and Airborne Instrumentation for Astronomy VI, Proc. SPIE, 9908, 99082S

Goodrich, R. W., \& Dahari, O. 1985, ApJ, 289, 342

Górny, S. K. 2014, A\&A, 570, A26

Górny, S. K., Chiappini, C., Stasińska, G., \& Cuisinier, F. 2009, A\&A, 500, 1089

Greenstein, J. L., \& Minkowski, R. 1964, ApJ, 140, 1601

Guerrero, M. A., \& De Marco, O. 2013, A\&A, 553, A126

Handler, G. 2003, in Planetary Nebulae: Their Evolution and Role in the Universe, eds. S. Kwok, M. Dopita, \& R. Sutherland, IAU Symp., 209, 237

Hanuschik, R. W. 2003, A\&A, 407, 1157

Herbig, G. H. 1995, ARA\&A, 33, 19
Hillwig, T. C., Margheim, S. J., De Marco, O., \& Frew, D. 2013, AAS Meeting Abstracts, 221, 249.09

Hillwig, T. C., Jones, D., De Marco, O., et al. 2016, ApJ, 832, 125

Hillwig, T. C., Frew, D., Jones, D., \& Crispo, D. 2017, AAS Meeting Abstracts, 229, 148.10

Hook, I. M., Jørgensen, I., Allington-Smith, J. R., et al. 2004, PASP, 116, 425

Hu, J. Y., \& Bibo, E. A. 1990, A\&A, 234, 435

Kramida, A., Ralchenko, Yu., Reader, J., \& NIST ASD Team 2017, NIST Atomic Spectra Database (ver. 5.5.1) (Gaithersburg, MD: National Institute of Standards and Technology) https://physics.nist.gov/asd

Kronberger, M., Parker, Q. A., Jacoby, G. H., et al. 2016, J. Phys. Conf. Ser., 728, 072012

Kudritzki, R. P., Simon, K. P., \& Méndez, R. H. 1981, The Messenger, 26, 7

Le Dû, P. 2017, L'Astronomie, 131, 46

Mendez, R. H. 1991, in Evolution of Stars: The Photospheric Abundance Connection, eds. G. Michaud, \& A. V. Tutukov, IAU Symp., 145, 375

Mendez, R. H., Kudritzki, R. P., Herrero, A., Husfeld, D., \& Groth, H. G. 1988, A\&A, 190, 113

Mendez, R. H., Kudritzki, R. P., \& Herrero, A. 1992, A\&A, 260, 329

Miller Bertolami, M. M. 2016, A\&A, 588, A25

Miszalski, B., Parker, Q. A., Acker, A., et al. 2008, MNRAS, 384, 525

Miszalski, B., Acker, A., Parker, Q. A., \& Moffat, A. F. J. 2009a, A\&A, 505, 249

Miszalski, B., Acker, A., Moffat, A. F. J., Parker, Q. A., \& Udalski, A. 2009b, A\&A, 496, 813

Miszalski, B., Jones, D., Rodríguez-Gil, P., et al. 2011, A\&A, 531, A158

Moore, C. E. 1945, Contributions from the Princeton University Observatory, 20,1

Napiwotzki, R., Tovmassian, G., Richer, M. G., et al. 2005, in Planetary Nebulae as Astronomical Tools, eds. R. Szczerba, G. Stasińska, \& S. K. Gorny, AIP Conf. Ser., 804, 173

Østensen, R. H., Silvotti, R., Charpinet, S., et al. 2010, MNRAS, 409, 1470

Parker, Q. A., Acker, A., Frew, D. J., et al. 2006, MNRAS, 373, 79

Parker, Q. A., Frew, D. J., Acker, A., \& Miszalski, B. 2012, IAU Symp., 283, 9

Sabin, L., Parker, Q. A., Corradi, R. L. M., et al. 2014, MNRAS, 443, 3388

Schonberner, D. 1989, in Planetary Nebulae, ed. S. Torres-Peimbert, IAU Symp., 131,463

Shappee, B. J., Prieto, J. L., Grupe, D., et al. 2014, ApJ, 788, 48

Simpson, J. P., Sellgren, K., Ramirez, S., Cotera, A., \& An, D. 2015, IAU General Assembly, 22, 2251870

Sota, A., Maíz Apellániz, J., Walborn, N. R., et al. 2011, ApJS, 193, 24

Sota, A., Maíz Apellániz, J., Morrell, N. I., et al. 2014, ApJS, 211, 10

Tovmassian, G. H., Napiwotzki, R., Richer, M. G., et al. 2004, ApJ, 616, 485

Weidmann, W. A., \& Gamen, R. 2011a, A\&A, 531, A172 (Paper II)

Weidmann, W. A., \& Gamen, R. 2011b, A\&A, 526, A6 (Paper I)

Weidmann, W. A., Méndez, R. H., \& Gamen, R. 2015, A\&A, 579, A86 (Paper III) 


\section{Appendix A: Atlas of spectra}

Table A.1. Details of the spectra of CSPN observed with the INT.

\begin{tabular}{llccc}
\hline \hline PN G & Name & Date & PA [ $\left.{ }^{\circ}\right]$ & Exptime [s] \\
\hline $038.4-03.3$ & K 4-19 & $07-28-2014$ & 205 & $3 \times 900$ \\
$039.5-02.7$ & M 2-47 & $09-01-2016$ & 0 & $3 \times 2100$ \\
$042.0+05.4$ & K 3-14 & $07-12-2016$ & 300 & $3 \times 1800$ \\
$044.3-05.6$ & K 3-36 & $09-01-2016$ & 57 & $3 \times 2100$ \\
$047.1+04.1$ & K 3-21 & $07-13-2016$ & 300 & $2 \times 1800$ \\
$054.4-02.5$ & M 1-72 & $05-21-2015$ & 305 & $3 \times 2100$ \\
$055.6+02.1$ & He 1-2 & $07-13-2016$ & 290 & $3 \times 1800$ \\
$107.6-13.3$ & Vy 2-3 & $08-17-2016$ & 310 & $2 \times 1200$ \\
$167.4-09.1$ & K 3-66 & $01-10-2014$ & 120 & $3 \times 1800$ \\
$184.0-02.1$ & M 1-5 & $11-12-2016$ & 74 & $1 \times 1200$ \\
$199.4+14.3$ & PM 1-29 & $02-25-2016$ & 64 & $3 \times 1200$ \\
- & K 4-50 & $11-08-2014$ & 0 & $2 \times 1500$ \\
\hline
\end{tabular}

Notes. The PA and exptime columns are the same as in Table A.2. The object K 4-50 (PK 208+01.1) was classified as possible PN by Acker et al. (1992).

Table A.2. Details of the spectra of CSPNs observed with GMOS.

\begin{tabular}{|c|c|c|c|c|c|c|c|}
\hline PN G & Name & Programme ID & Grating & Spectral range $[\AA]$ & $\mathrm{PA}\left[{ }^{\circ}\right]$ & Exptime [s] & $\operatorname{Bin}[p x]$ \\
\hline $000.2-01.9$ & M 2-19 & GS-2008B-Q-65 & B1200 & $4090-5540$ & 90 & $3 \times 1336$ & $2 \times 2$ \\
\hline $001.7-04.6$ & H $1-56$ & GS-2016A-Q-74 & B1200 & $3950-5500$ & 76 & $3 \times 500^{\dagger}$ & $1 \times 1$ \\
\hline $001.8-02.0$ & PHR J1757-2824 & GS-2009A-Q-35 & B1200 & $4100-5530$ & 120 & $2 \times 1950^{\dagger}$ & $1 \times 2$ \\
\hline $001.8-03.7$ & PHR J1804-2913 & GS-2009A-Q-35 & B1200 & $4100-5530$ & 90 & $1 \times 1200^{\dagger}$ & $1 \times 2$ \\
\hline $003.3-04.6$ & Ap $1-12$ & GS-2016A-Q-101 & $\mathrm{B} 600$ & $3500-6260$ & 71 & $3 \times 800$ & $1 \times 1$ \\
\hline $003.5-02.4$ & IC 4673 & GS-2011A-Q-65 & B1200 & $3830-5200$ & 125 & $1 \times 1800$ & $1 \times 2$ \\
\hline $005.0+03.0$ & Pe 1-9 & GS-2009A-Q-35 & B1200 & $4100-5530$ & 45 & $1 \times 2700^{\dagger}$ & $1 \times 2$ \\
\hline $007.8-04.4$ & H $1-65$ & GS-2011A-Q-65 & B1200 & $4000-5200$ & 270 & $1 \times 900$ & $1 \times 2$ \\
\hline $011.3-09.4$ & H $2-48$ & GS-2016A-Q-101 & B600 & $3500-6260$ & 90 & $3 \times 900^{\dagger}$ & $1 \times 1$ \\
\hline $012.1-11.2$ & PPA J1855-2328 & GS-2015A-Q-98 & B600 & $3750-6750$ & 70 & $2 \times 1000$ & $2 \times 2$ \\
\hline $013.1+05.0$ & Sa 3-96 & GS-2015AQ-98 & B600 & $3660-6750$ & 55 & $3 \times 600^{\dagger}$ & $2 \times 2$ \\
\hline $014.8-08.4$ & SB 20 & GS-2015AQ-98 & B600 & $3660-6750$ & 90 & $3 \times 1200$ & $2 \times 2$ \\
\hline $016.4-01.9$ & M 1-46 & GS-2016A-Q-101 & B600 & $3500-6260$ & 64 & $3 \times 600$ & $1 \times 1$ \\
\hline $017.6-10.2$ & A 51 & GS-2016A-Q-101 & B600 & $3500-6260$ & 239 & $3 \times 1000$ & $1 \times 1$ \\
\hline $020.7-05.9$ & Sa $1-8$ & GS-2016A-Q-101 & B 600 & $3500-6260$ & 239 & $3 \times 900$ & $1 \times 1$ \\
\hline $034.3+06.2$ & K 3-5 & GN-2016A-Q-97 & B600 & $3500-6000$ & 309 & $3 \times 900^{\dagger}$ & $2 \times 2$ \\
\hline 036.4-01.9 & IPHAS $190438^{a}$ & GN-2015A-Q-405 & B600 & $3850-6600$ & 0 & $3 \times 600$ & $2 \times 2$ \\
\hline $039.0-04.0$ & IPHAS $191716^{b}$ & GN-2015A-Q-405 & B600 & $3850-6600$ & 123 & $1 \times 600^{\dagger}$ & $2 \times 2$ \\
\hline $051.3+01.8$ & PM 1-295 & GN-2016A-Q-97 & B600 & $3500-6000$ & 282 & $3 \times 1000$ & $2 \times 2$ \\
\hline $052.2+07.6$ & K 4-10 & GN-2016A-Q-97 & B600 & $3500-6000$ & 266 & $3 \times 1000$ & $2 \times 2$ \\
\hline $060.0-04.3$ & A 68 & GN-2015A-Q-405 & B600 & $3850-6600$ & 120 & $3 \times 1000^{\dagger}$ & $2 \times 2$ \\
\hline $066.8+02.9$ & IPHAS $194751^{c}$ & GN-2015A-Q-405 & B600 & $3850-6600$ & 80 & $3 \times 1000$ & $2 \times 2$ \\
\hline $068.7+14.8$ & Sp 4-1 & GN-2016A-Q-97 & B600 & $3500-6000$ & 37 & $3 \times 900$ & $2 \times 2$ \\
\hline $075.7+35.8$ & $\mathrm{Sa} 4-1$ & GN-2015A-Q-405 & B600 & $3850-6600$ & 280 & $2 \times 1200$ & $2 \times 2$ \\
\hline $075.9+11.6$ & AMU 1 & GN-2012A-Q-124 & B1200 & $4000-5500$ & 147 & $4 \times 360$ & $2 \times 2$ \\
\hline $078.5+18.7$ & A 50 & GN-2016A-Q-97 & B600 & $3500-6000$ & 215 & $2 \times 1000^{\dagger}$ & $2 \times 2$ \\
\hline $135.9+55.9$ & TS 01 & GN-2006A-Q-82 & B1200 & $3800-5000$ & 25 & $8 \times 700$ & $2 \times 2$ \\
\hline $211.2-03.5$ & M 1-6 & GS-2015B-Q-103 & B600 & $3500-6400$ & 258 & $5 \times 900$ & $2 \times 2$ \\
\hline $234.9-01.4$ & M 1-14 & GS-2015B-Q-103 & B600 & $3500-6400$ & 0 & $2 \times 770$ & $2 \times 2$ \\
\hline $236.9+08.6$ & PHR J0809-1650 & GS-2015B-Q-103 & B600 & $3500-6400$ & 294 & $4 \times 770$ & $2 \times 2$ \\
\hline $238.0+34.8$ & A 33 & GS-2015B-Q-103 & B600 & $3500-6400$ & 207 & $5 \times 450$ & $2 \times 2$ \\
\hline $249.8+07.1$ & PHR J0834-2819 & GS-2016B-Q-65 & B1200 & $4000-5500$ & 50 & $3 \times 950$ & $2 \times 2$ \\
\hline
\end{tabular}

Notes. The PA column indicates the slit position angle in degrees, "exptime" is the integration time (underexposed spectra is denoted by ${ }^{\dagger}$ ), and "bin" is the binning applied to the CCDs. ${ }^{(a)}$ IPHASX J190438.6+021424, ${ }^{(b)}$ IPHASX J191716.4+033447, ${ }^{(c)}$ IPHASX J194751.9+311818, (d) 4500-5900 ̊, and ${ }^{(e)} 4500-5900 \AA$. 
Table A.2. continued

\begin{tabular}{|c|c|c|c|c|c|c|c|}
\hline PN G & Name & Programme ID & Grating & Spectral range $[\AA]$ & $\mathrm{PA}\left[{ }^{\circ}\right]$ & Exptime [s] & $\operatorname{Bin}[\mathrm{px}]$ \\
\hline $250.3-05.4$ & PHR J0745-3535 & GS-2015B-Q-103 & B600 & $3500-6400$ & 265 & $4 \times 750$ & $2 \times 2$ \\
\hline $258.5-01.3$ & $\mathrm{RCW} 24$ & GS-2008B-Q-65 & B1200 & $4090-5540$ & 3 & $1 \times 2400$ & $2 \times 2$ \\
\hline $273.6+06.1$ & HbDs 1 & GS-2015B-Q-103 & B600 & $3500-6400$ & 0 & $3 \times 400$ & $2 \times 2$ \\
\hline $281.0-05.6$ & IC 2501 & GS-2015B-Q-103 & $\mathrm{B} 600$ & $3500-6400$ & 210 & $3 \times 900$ & $2 \times 2$ \\
\hline $285.6-02.7$ & He $2-47$ & GS-2011A-Q-65 & B1200 & $3900-5200^{d}$ & 90 & $1 \times 1200$ & $1 \times 2$ \\
\hline $285.7-14.9$ & IC 2448 & GS-2015B-Q-103 & $\mathrm{B} 600$ & $3500-6400$ & 61 & $1 \times 770$ & $2 \times 2$ \\
\hline $288.7+08.1$ & ESO 216-2 & GS-2015AQ-98 & B600 & $3750-6750$ & 90 & $3 \times 1000$ & $2 \times 2$ \\
\hline $302.2-03.1$ & PHR J1244-6601 & GS-2015AQ-98 & B600 & $4000-6750$ & 183 & $3 \times 1000^{\dagger}$ & $2 \times 2$ \\
\hline $310.3+24.7$ & Lo 8 & GS-2016A-Q-101 & B600 & $3500-6260$ & 277 & $3 \times 600$ & $1 \times 1$ \\
\hline $312.6-01.8$ & He 2-107 & GS-2011A-Q-65 & B1200 & $3900-5200^{e}$ & 90 & $1 \times 1800$ & $1 \times 2$ \\
\hline $315.4-08.4$ & PHR J1510-6754 & GS-2012A-Q-85 & B1200 & $4000-5450$ & 337 & $2 \times 600^{\dagger}$ & $2 \times 2$ \\
\hline $315.7+05.5$ & LoTr 8 & GS-2011A-Q-65 & B1200 & $3830-5200$ & 134 & $1 \times 2400$ & $1 \times 2$ \\
\hline $317.2+08.6$ & PHR J1424-5138 & GS-2015A-Q-98 & $\mathrm{B} 600$ & $3750-6750$ & 282 & $3 \times 600$ & $2 \times 2$ \\
\hline $323.6-04.5$ & WKK136-337 & GS-2011A-Q-65 & B1200 & $3800-5200$ & 90 & $1 \times 900$ & $1 \times 2$ \\
\hline $323.9+02.4$ & He $2-123$ & GS-2016A-Q-74 & B1200 & $3950-5500$ & 179 & $3 \times 500$ & $1 \times 1$ \\
\hline $325.8-12.8$ & He 2-182 & GS-2016A-Q-101 & B600 & $3500-6260$ & 310 & $3 \times 900$ & $1 \times 1$ \\
\hline $326.0-06.5$ & He $2-151$ & GS-2016A-Q-101 & $\mathrm{B} 600$ & $3500-6260$ & 322 & $3 \times 600$ & $1 \times 1$ \\
\hline $326.4+07.0$ & NeVe 3-2 & GS-2011A-Q-65 & B1200 & $3830-5200$ & 225 & $1 \times 1200$ & $1 \times 2$ \\
\hline $329.0+01.9$ & Sp 1 & GS-2011A-Q-91 & B1200 & $4000-5460$ & 90 & $1 \times 480$ & $2 \times 2$ \\
\hline $329.5+01.7$ & VBRC 7 & GS-2015A-Q-98 & B600 & $4000-6750$ & 320 & $3 \times 1200$ & $2 \times 2$ \\
\hline $331.4-03.5$ & He $2-162$ & GS-2016A-Q-101 & B600 & $3500-6260$ & 270 & $3 \times 900$ & $1 \times 1$ \\
\hline $332.8-16.4$ & HaTr 6 & GS-2015A-Q-98 & B600 & $4000-5500$ & 270 & $3 \times 1000^{\dagger}$ & $2 \times 2$ \\
\hline $338.1-08.3$ & NGC 6326 & GS-2009A-Q-35 & B1200 & $4510-5960$ & 90 & $1 \times 1800^{\dagger}$ & $1 \times 2$ \\
\hline $349.3-04.2$ & Lo 16 & GS-2009A-Q-35 & B1200 & $4510-5960$ & 100 & $1 \times 1800$ & $1 \times 2$ \\
\hline $352.1-02.6$ & PHR J1736-3659 & GS-2011A-Q-65 & B1200 & $3830-5200$ & 90 & $1 \times 900$ & $1 \times 2$ \\
\hline $353.7+06.3$ & M 2-7 & GS-2016A-Q-101 & $\mathrm{B} 600$ & $3500-6260$ & 90 & $3 \times 900$ & $1 \times 1$ \\
\hline $354.5-03.9$ & SAB 41 & GS-2008B-Q-65 & B600 & $4260-7030$ & 149 & $1 \times 1800^{\dagger}$ & $2 \times 2$ \\
\hline $355.3-03.2$ & PPA J1747-3435 & GS-2009A-Q-35 & B1200 & $4100-5530$ & 135 & $1 \times 2400^{\dagger}$ & $1 \times 2$ \\
\hline $355.6-02.3$ & PHR J1744-3355 & GS-2009A-Q-35 & B1200 & $4100-5530$ & 168 & $2 \times 1950^{\dagger}$ & $1 \times 2$ \\
\hline $357.0-04.4$ & PHR J1756-3342 & GS-2009A-Q-35 & B1200 & $4100-5530$ & 180 & $1 \times 2400$ & $1 \times 2$ \\
\hline $357.1-05.3$ & BMP J1800-3408 & GS-2009A-Q-35 & B1200 & $4100-5530$ & 35 & $1 \times 2400$ & $1 \times 2$ \\
\hline $357.6-03.3$ & Н $2-29$ & GS-2009A-Q-35 & B1200 & $4100-5530$ & 120 & $2 \times 1950^{\dagger}$ & $1 \times 2$ \\
\hline $358.7-03.0$ & K 6-34 & GS-2009A-Q-35 & B1200 & $4100-5530$ & 90 & $1 \times 2400$ & $1 \times 2$ \\
\hline $359.1-02.3$ & M 3-16 & GS-2008B-Q-65 & B1200 & $4090-5540$ & 90 & $4 \times 1200$ & $1 \times 2$ \\
\hline
\end{tabular}


Table A.3. Spectral classification and key lines of CSPNs sample.

\begin{tabular}{|c|c|c|c|c|c|c|c|c|c|c|c|}
\hline$N$ & Name & S.T. & $\begin{array}{l}\text { He II } \\
4542\end{array}$ & $\begin{array}{l}\text { He II } \\
4686\end{array}$ & $\begin{array}{l}\text { He II } \\
5412 \\
\end{array}$ & $\begin{array}{l}\text { He I } \\
4471\end{array}$ & $\begin{array}{c}\mathrm{H} \beta \\
4861\end{array}$ & $\begin{array}{c}\mathrm{N} \mathrm{v} \\
4603-19\end{array}$ & $\begin{array}{c}\text { C IV } \\
5801-12\end{array}$ & N.S. & Fig. \\
\hline 1 & A 33 & DAO & A & $\mathrm{A}$ & A & - & $\mathrm{A}$ & - & - & $\mathrm{y}$ & A. 19 \\
\hline 2 & A 50 & $\mathrm{O}(\mathrm{H}) \mathrm{III}-\mathrm{V}$ & - & $\mathrm{A}$ & - & - & $\mathrm{A}$ & - & - & $\mathrm{y}$ & A. 16 \\
\hline 3 & A 51 & $\mathrm{O}(\mathrm{H}) 3-5 \mathrm{Vz}$ & A & $\mathrm{A}$ & $\mathrm{A}$ & - & $\mathrm{A}$ & $\mathrm{E}$ & - & $\mathrm{n}$ & A. 5 \\
\hline 4 & A 68 & H-rich & - & - & - & - & - & - & - & $\mathrm{y}$ & A. 20 \\
\hline 5 & AMU 1 & $\mathrm{O}(\mathrm{H}) 3 \mathrm{Vz}$ & A & A & A & - & $\mathrm{A}$ & A & N/D & $\mathrm{y}$ & A. 1 \\
\hline 6 & Ap 1-12 & B0 I-III & - & - & A & A & - & - & A & $\mathrm{n}$ & A.13 \\
\hline 7 & BMP J1800-3408 & $\mathrm{O}(\mathrm{H}) 4-5 \mathrm{Vz}$ & A & $\mathrm{A}$ & A & - & $\mathrm{A}$ & - & $\mathrm{N} / \mathrm{D}$ & $\mathrm{n}$ & A. 6 \\
\hline 8 & ESO 216-2 & $\mathrm{O}(\mathrm{H}) 4-5 \mathrm{~V}$ & $\mathrm{~A}$ & A & $\mathrm{A}$ & - & A & - & $\mathrm{E}$ & $\mathrm{y}$ & A. 5 \\
\hline 9 & H 1-56 & $\mathrm{O} ?$ & A? & - & A? & - & - & - & $\mathrm{N} / \mathrm{D}$ & $\mathrm{n}$ & A. 22 \\
\hline 10 & H $1-65$ & $\mathrm{O}(\mathrm{H}) 8-9 \mathrm{I}$ & $\mathrm{A}$ & $\mathrm{E}$ & N/D & A & - & - & $\mathrm{N} / \mathrm{D}$ & $\mathrm{n}$ & A. 10 \\
\hline 11 & Н 2-29 & $\mathrm{O}(\mathrm{H})$ & A & A & $\mathrm{A}$ & - & A & - & $\mathrm{N} / \mathrm{D}$ & $\mathrm{n}$ & A. 15 \\
\hline 12 & H 2-48 & O III-V & $\mathrm{A}$ & A & $\mathrm{A}$ & - & - & - & A & $\mathrm{n}$ & A. 18 \\
\hline 13 & HaTr 6 & H-rich & - & - & - & - & A & - & - & $\mathrm{n}$ & A. 20 \\
\hline 14 & HbDs 1 & $\mathrm{O}(\mathrm{H}) 3 \mathrm{Vz}$ & A & $\mathrm{A}$ & $\mathrm{A}$ & - & $\mathrm{A}$ & $\mathrm{E}$ & $\mathrm{E}$ & $\mathrm{y}$ & A. 1 \\
\hline 15 & He $1-2$ & O9-B0 I & - & - & - & A & - & - & - & $\mathrm{n}$ & A. 9 \\
\hline 16 & He $2-47$ & $\mathrm{O}(\mathrm{H}) 7-9 \mathrm{I}$ & A & $\mathrm{E}$ & $\mathrm{A}$ & $?$ & - & - & A & $\mathrm{n}$ & A. 10 \\
\hline 17 & He $2-107$ & $\mathrm{O}(\mathrm{H}) 4 \mathrm{Ifc}$ & A & $\mathrm{E}$ & A & A & - & A? & $\mathrm{A}+\mathrm{E}$ ? & $\mathrm{y}$ & A. 4 \\
\hline 18 & He $2-123$ & O3-4 & A & - & - & - & - & A? & N/D & n & A. 18 \\
\hline 19 & He $2-151$ & B0 & $\mathrm{A}$ & A & $\mathrm{A}$ & A & $\mathrm{A}$ ? & - & A & $\mathrm{n}$ & A. 14 \\
\hline 20 & He 2-162 & B0 I-III & - & $\mathrm{A}$ & - & A & $\mathrm{A}$ & - & A & $\mathrm{n}$ & A.13 \\
\hline 21 & He $2-182$ & $\mathrm{O}(\mathrm{H}) 4-8 \mathrm{III}-\mathrm{V}$ & $\mathrm{A}$ & $\mathrm{A}$ & $\mathrm{A}$ & - & - & - & A & $\mathrm{n}$ & A. 11 \\
\hline 22 & IC 2448 & $\mathrm{O}(\mathrm{H}) 3 \mathrm{III}-\mathrm{V}$ & A & A & A & - & A & $\mathrm{A}$ & $\mathrm{E}$ & $\mathrm{y}$ & A. 4 \\
\hline 23 & IC 2501 & O3-6 & $\mathrm{A}$ & - & A? & - & - & A? & $\mathrm{E}$ & $\mathrm{n}$ & A. 8 \\
\hline 24 & IC 4673 & $\mathrm{O}(\mathrm{H}) 8$ & - & - & N/D & A & A & - & $\mathrm{N} / \mathrm{D}$ & $\mathrm{y}$ & A. 10 \\
\hline 25 & IPHAS 190438 & $\mathrm{~B}[\mathrm{e}]$ & - & - & - & - & $\mathrm{E}$ & - & - & $\mathrm{n}$ & A. 14 \\
\hline 26 & IPHAS 191716 & $\mathrm{O}(\mathrm{H}) \mathrm{III}-\mathrm{V}(\mathrm{e})$ & A & $\mathrm{A}$ & A? & - & $\mathrm{A}$ & - & - & $\mathrm{y}$ & A. 16 \\
\hline 27 & IPHAS 194751 & B0 I & - & - & - & A & A? & - & - & n & A. 12 \\
\hline 28 & K 3-5 & emission line? & - & $\mathrm{E}$ ? & $\mathrm{E} ?$ & $\mathrm{E} ?$ & $\mathrm{E}$ ? & - & $\mathrm{E} ?$ & $\mathrm{n}$ & A. 24 \\
\hline 29 & K 3-14 & O8-9 & - & $\mathrm{A}$ & A & - & - & - & A & $\mathrm{n}$ & A.9 \\
\hline 30 & K 3-21 & cont. & - & - & - & - & - & - & - & $\mathrm{n}$ & A. 23 \\
\hline 31 & K $3-36$ & $\mathrm{O} ?$ & - & - & - & - & - & A? & $\mathrm{E}$ & $\mathrm{n}$ & A. 22 \\
\hline 32 & K 3-66 & $\mathrm{O}$ & A & - & - & - & - & - & $\mathrm{A}$ & $\mathrm{n}$ & A. 18 \\
\hline 33 & K 4-10 & O3-4 & A & - & A? & - & - & A & $\mathrm{E}$ ? & $\mathrm{n}$ & A. 4 \\
\hline 34 & K 4-19 & B1 I & - & - & - & $\mathrm{A}$ & A & - & - & $\mathrm{n}$ & A.13 \\
\hline 35 & $\mathrm{~K} 4-50$ & $\mathrm{~B}$ ? & - & - & - & A & - & - & - & $\mathrm{n}$ & A. 14 \\
\hline 36 & K 6-34 & $\mathrm{O}(\mathrm{H}) 4-6$ & $\mathrm{~A}$ & - & $\mathrm{A}$ & - & $\mathrm{A}$ & - & $\mathrm{N} / \mathrm{D}$ & $\mathrm{y}$ & A. 7 \\
\hline 37 & Lo 8 & $\mathrm{O}(\mathrm{H}) 3 \mathrm{Vz}$ & $\mathrm{A}$ & A & $\mathrm{A}$ & - & $\mathrm{A}$ & $\mathrm{A}$ & $\mathrm{E}$ & $\mathrm{y}$ & A. 2 \\
\hline 38 & Lo 16 & $\mathrm{O}(\mathrm{H}) 3-4 \mathrm{Vz}((\mathrm{fc}))$ & A & A & $\mathrm{A}$ & - & A & $\mathrm{A}$ & $\mathrm{E}$ & $\mathrm{y}$ & A. 3 \\
\hline 39 & LoTr 8 & [WR] & - & $\mathrm{E}$ & - & - & - & - & - & $\mathrm{n}$ & A. 24 \\
\hline 40 & M 1-5 & emission line? & - & $\mathrm{E}$ ? & - & - & - & - & $\mathrm{E}$ ? & $\mathrm{n}$ & A. 24 \\
\hline 41 & M 1-6 & O6-9 I & A & - & A? & - & - & - & $\mathrm{A}$ & $\mathrm{n}$ & A. 8 \\
\hline 42 & M 1-14 & $\mathrm{O}(\mathrm{H}) 7-9$ & A & - & $\mathrm{A}$ & - & - & - & A & $\mathrm{n}$ & A. 15 \\
\hline 43 & M 1-46 & $\mathrm{O}(\mathrm{H}) 7 \mathrm{I}(\mathrm{fc})$ & A & $\mathrm{E}$ & $\mathrm{A}$ & $\mathrm{A}$ & - & - & A & $\mathrm{y}$ & A. 7 \\
\hline 44 & M 1-72 & cont. & - & - & - & - & - & - & - & $\mathrm{n}$ & A. 23 \\
\hline 45 & M 2-7 & $\mathrm{O}$ & A? & A & A & - & - & - & - & $\mathrm{n}$ & A. 18 \\
\hline 46 & M 2-19 & $\mathrm{O}(\mathrm{H}) 6-8 \mathrm{III}-\mathrm{V}$ & $\mathrm{A}$ & A & $\mathrm{A}$ & - & $\mathrm{A}$ & - & $\mathrm{N} / \mathrm{D}$ & $\mathrm{n}$ & A. 5 \\
\hline 47 & M 2-47 & O3-6 & A & $\mathrm{E}$ ? & A & - & - & A? & $\mathrm{E}$ & $\mathrm{n}$ & A. 8 \\
\hline 48 & M 3-16 & $\mathrm{O}(\mathrm{H}) 4-6$ III-V & A & A & A & $\mathrm{A}$ & $\mathrm{A}$ & - & $\mathrm{N} / \mathrm{D}$ & $\mathrm{n}$ & A. 7 \\
\hline 49 & NeVe 3-2 & $\mathrm{O}(\mathrm{H}) 4 \mathrm{Vz}$ & A & A & N/D & - & A & A? & N/D & $\mathrm{y}$ & A.6 \\
\hline 50 & NGC 6326 & $\mathrm{O}(\mathrm{H}) 5-8((\mathrm{fc}))$ & - & A & A & A? & A & - & $\mathrm{E} ?$ & $\mathrm{y}$ & A. 11 \\
\hline 51 & Pe $1-9$ & $\mathrm{O}(\mathrm{H})$ & A? & $\mathrm{A}$ & $\mathrm{A}$ & A? & A & A? & $\mathrm{N} / \mathrm{D}$ & $\mathrm{y}$ & A. 17 \\
\hline 52 & PHR J0745-3535 & cont. & - & - & - & - & - & - & - & $\mathrm{y}$ & A. 23 \\
\hline 53 & PHR J0809-1650 & $\mathrm{O}(\mathrm{H}) \mathrm{III}-\mathrm{V}$ & A & $\mathrm{A}$ & $\mathrm{A}$ & - & $\mathrm{A}$ & - & - & $\mathrm{y}$ & A. 11 \\
\hline 54 & PHR J0834-2819 & DAO & - & $\mathrm{A}$ & - & - & $\mathrm{A}$ & - & $\mathrm{N} / \mathrm{D}$ & $\mathrm{y}$ & A. 19 \\
\hline 55 & PHR J1244-6601 & H-rich & - & - & - & - & $\mathrm{A}$ & - & - & $\mathrm{n}$ & A. 20 \\
\hline
\end{tabular}

Notes. The N.S. column indicates whether or not it was possible to subtract the nebular component. The letters A and E indicate whether the ion was in absorption or emission, respectively. An undetected ion was denoted (-), and N/D means no data available in the corresponding spectral range. 
Table A.3. continued

\begin{tabular}{|c|c|c|c|c|c|c|c|c|c|c|c|}
\hline $\mathrm{N}$ & Name & S.T. & $\begin{array}{l}\text { He II } \\
4542\end{array}$ & $\begin{array}{l}\text { He II } \\
4686\end{array}$ & $\begin{array}{l}\text { He II } \\
5412\end{array}$ & $\begin{array}{l}\mathrm{He}_{\mathrm{I}} \\
4471\end{array}$ & $\begin{array}{c}\mathrm{H} \beta \\
4861\end{array}$ & $\begin{array}{c}\mathrm{N} \mathrm{v} \\
4603-19\end{array}$ & $\begin{array}{c}\text { C IV } \\
5801-12\end{array}$ & N.S. & Fig. \\
\hline 56 & PHR J1424-5138 & $\mathrm{O}(\mathrm{H}) 3 \mathrm{I}$ & $\mathrm{A}$ & $\mathrm{N} / \mathrm{D}$ & A & - & A & A & $\mathrm{E}$ & $\mathrm{y}$ & A. 2 \\
\hline 57 & PHR J1510-6754 & $\mathrm{O}(\mathrm{H}) \mathrm{e}$ & $\mathrm{E}$ & $\mathrm{E}$ & $\mathrm{E}$ & $\mathrm{E}$ & $\mathrm{E}$ & - & $\mathrm{N} / \mathrm{D}$ & $\mathrm{y}$ & A. 16 \\
\hline 58 & PHR J1736-3659 & $\mathrm{O}(\mathrm{H}) 8$ If & $\mathrm{A}$ & $\mathrm{E}$ & $\mathrm{N} / \mathrm{D}$ & $\mathrm{A}$ & A & - & $\mathrm{N} / \mathrm{D}$ & $\mathrm{y}$ & A. 10 \\
\hline 59 & PHR J1744-3355 & H-rich & - & - & - & - & $\mathrm{A}$ & - & $\mathrm{N} / \mathrm{D}$ & $\mathrm{y}$ & A. 20 \\
\hline 60 & PHR J1756-3342 & $\mathrm{O}(\mathrm{H}) 3-4 \mathrm{Vz}$ & $\mathrm{A}$ & A & A & - & A & A & $\mathrm{N} / \mathrm{D}$ & $\mathrm{y}$ & A. 3 \\
\hline 61 & PHR J1757-2824 & $\mathrm{O}(\mathrm{H})$ & - & $\mathrm{A}$ & A & - & A & - & $\mathrm{N} / \mathrm{D}$ & $\mathrm{y}$ & A. 17 \\
\hline 62 & PHR J1804-2913 & $\mathrm{O}(\mathrm{H})$ & - & A & - & - & A & - & $\mathrm{N} / \mathrm{D}$ & $\mathrm{n}$ & A.17 \\
\hline 63 & PM 1-29 & B0.5 I & - & - & - & A & A & - & - & $\mathrm{n}$ & A.12 \\
\hline 64 & PM 1-295 & $\mathrm{O}(\mathrm{H}) 9 \mathrm{I}$ & - & - & - & $\mathrm{A}$ & $\mathrm{A}$ & - & - & $\mathrm{n}$ & A. 12 \\
\hline 65 & PPA J1747-3435 & $\mathrm{O}(\mathrm{H})$ & $\mathrm{A}$ & A & A & - & A & - & $\mathrm{N} / \mathrm{D}$ & $\mathrm{n}$ & A. 15 \\
\hline 66 & PPA J1855-2328 & $\mathrm{O}(\mathrm{H}) 6 \mathrm{III}-\mathrm{V}$ & $\mathrm{A}$ & $\mathrm{A}$ & $\mathrm{A}$ & - & $\mathrm{A}$ & - & - & $\mathrm{n}$ & A. 7 \\
\hline 67 & RCW 24 & DA & - & - & - & - & A & - & $\mathrm{N} / \mathrm{D}$ & $\mathrm{y}$ & A. 19 \\
\hline 68 & Sa $1-8$ & $\mathrm{O}(\mathrm{H}) 4-8 \mathrm{III}$ & $\mathrm{A}$ & $\mathrm{A}$ & A & - & - & - & $\mathrm{E}$ & $\mathrm{n}$ & A. 11 \\
\hline 69 & Sa 3-96 & H-rich? & - & - & - & - & A & - & - & $\mathrm{y}$ & A. 21 \\
\hline 70 & $\mathrm{Sa} 4-1$ & $\mathrm{O}(\mathrm{H}) 3 \mathrm{~V}$ & A & $\mathrm{A}$ & A & - & A & $\mathrm{A}$ & $\mathrm{E}$ & $\mathrm{y}$ & A.1 \\
\hline 71 & SAB 41 & H-rich & - & - & - & - & $\mathrm{A}$ & - & - & $\mathrm{y}$ & A. 21 \\
\hline 72 & SB 20 & $\mathrm{O}(\mathrm{H}) 3-4$ & A & $\mathrm{N} / \mathrm{D}$ & A & - & A & $\mathrm{A}$ & $\mathrm{E}$ & y & A. 3 \\
\hline 73 & $\mathrm{Sp} 1$ & $\mathrm{O}(\mathrm{H}) 3-5(\mathrm{fc})$ & $\mathrm{A}$ & $\mathrm{A}+\mathrm{E}$ ? & A & - & A & $\mathrm{E} ?$ & $\mathrm{~N} / \mathrm{D}$ & $\mathrm{y}$ & A. 6 \\
\hline 74 & $\mathrm{Sp} 4-1$ & $\mathrm{O}$ ? & A? & - & - & - & - & - & $\mathrm{E}$ & $n$ & A. 22 \\
\hline 75 & TS 01 & $\mathrm{O}(\mathrm{H}) \mathrm{III}-\mathrm{V}$ & $\mathrm{A}$ & A & $\mathrm{N} / \mathrm{D}$ & - & - & - & $\mathrm{A}$ & $\mathrm{n}$ & A. 16 \\
\hline 76 & VBRC 7 & H-rich? & - & $\mathrm{N} / \mathrm{D}$ & - & - & $\mathrm{A}$ & - & - & $\mathrm{y}$ & A. 21 \\
\hline 77 & Vy $2-3$ & $\mathrm{O}(\mathrm{H}) 3-4 \mathrm{I}$ & A & $\mathrm{E} ?$ & $\mathrm{~A}$ & - & A? & A & $\mathrm{E}$ & $\mathrm{n}$ & A. 2 \\
\hline 78 & WKK 136-337 & $\mathrm{O}(\mathrm{H}) 3 \mathrm{III}-\mathrm{V}$ & A & $\mathrm{A}$ & $\mathrm{N} / \mathrm{D}$ & - & $\mathrm{A}$ & A & $\mathrm{N} / \mathrm{D}$ & $\mathrm{y}$ & A. 3 \\
\hline
\end{tabular}


W. Weidmann et al.: Towards an improvement in the spectral description of central stars of planetary nebulae
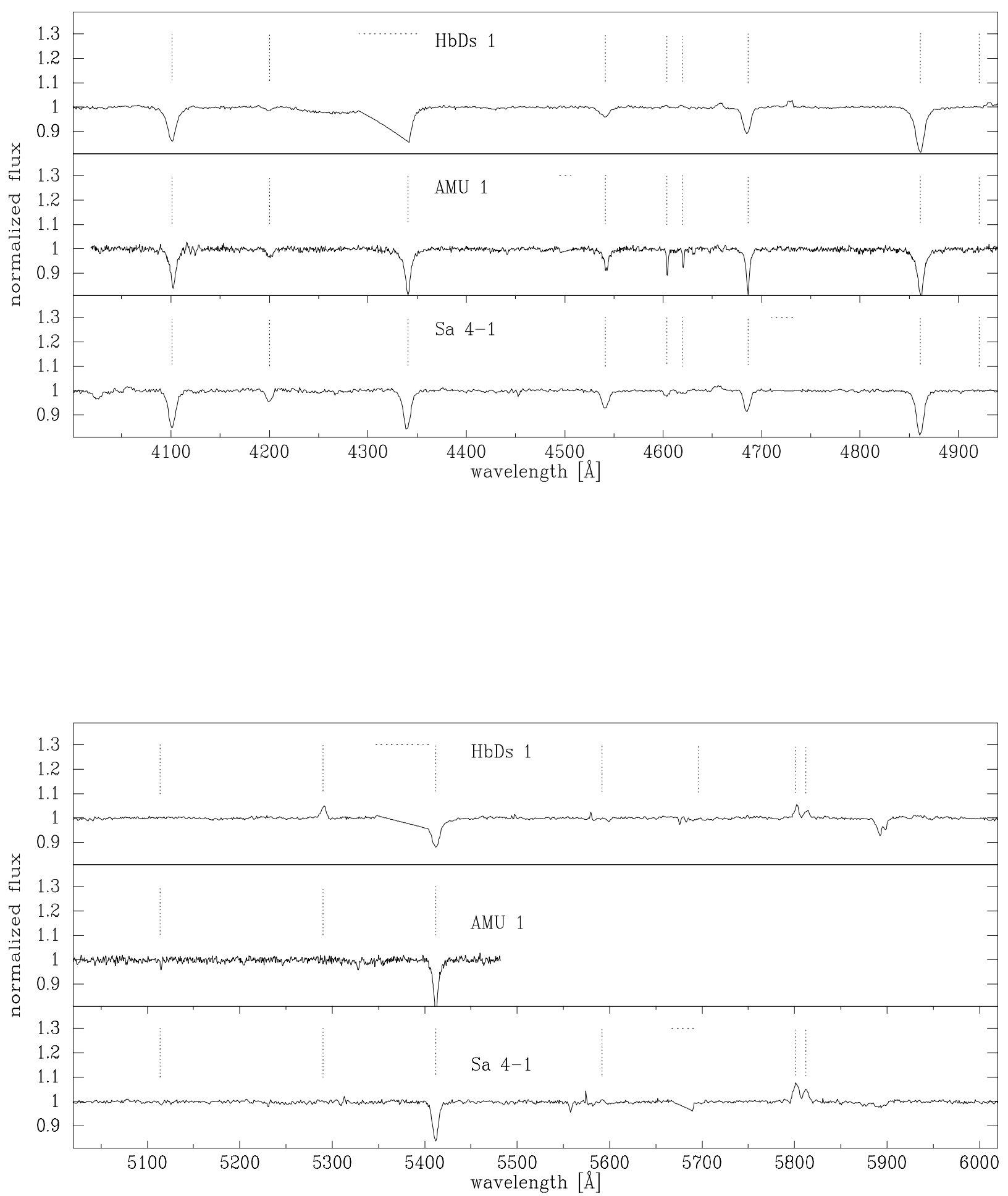

Fig. A.1. Normalized spectra of early O-type CSPN (see Table A.3). The interstellar absorption bands at $\lambda 4428$, the complex at 5780 and $5890-6$ are not indicated. The most important spectral features (absorption and emission) identified are: $\mathrm{H} \beta, \mathrm{H} \gamma, \mathrm{H} \delta, \mathrm{He}$ II $\lambda 4200,4542$, and $4686, \mathrm{~N} v$

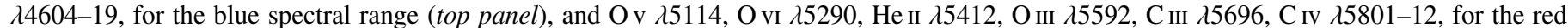
spectral range (bottom panel). 
A\&A 614, A135 (2018)
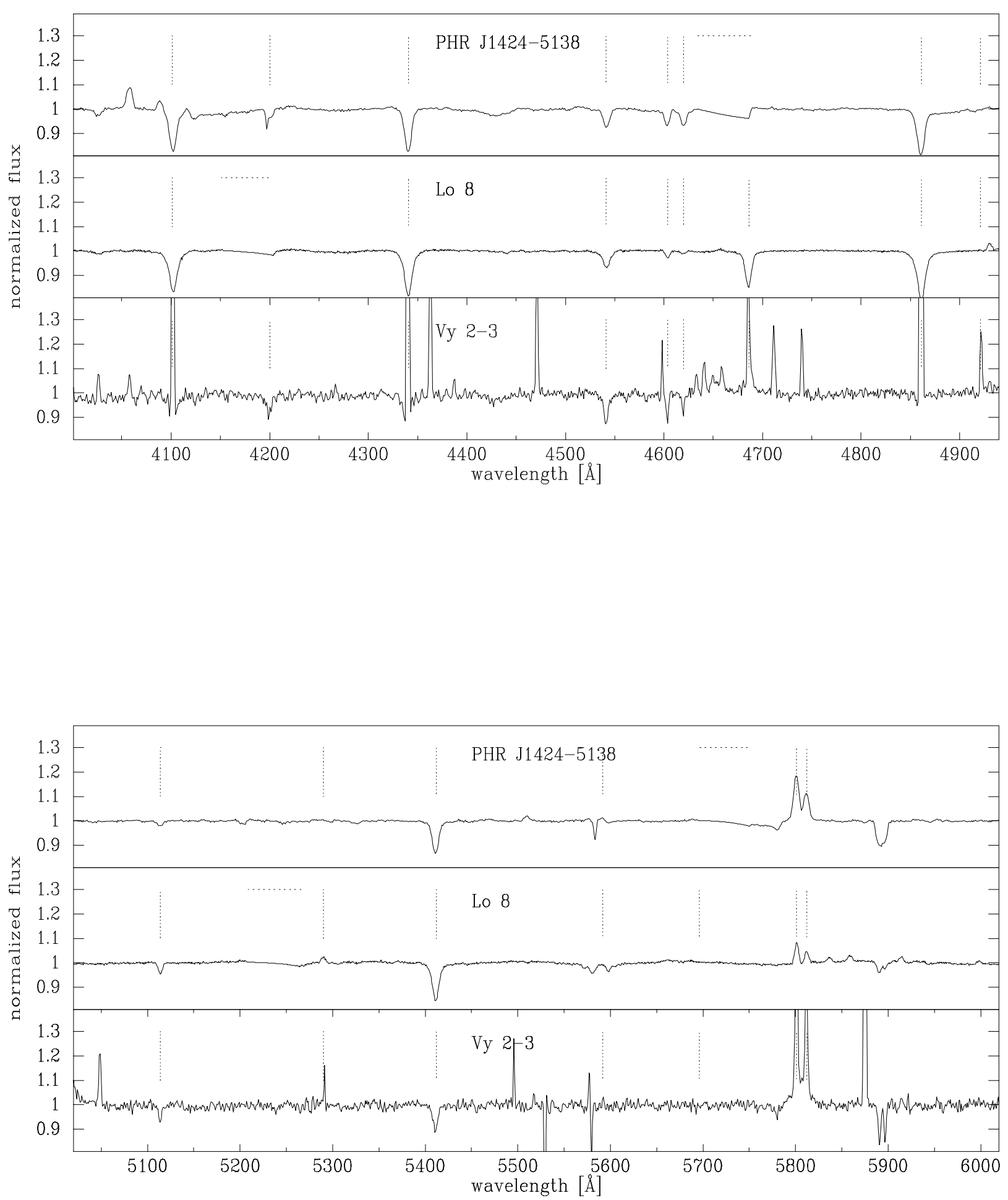

Fig. A.2. Same as Fig. A.1. 
W. Weidmann et al.: Towards an improvement in the spectral description of central stars of planetary nebulae
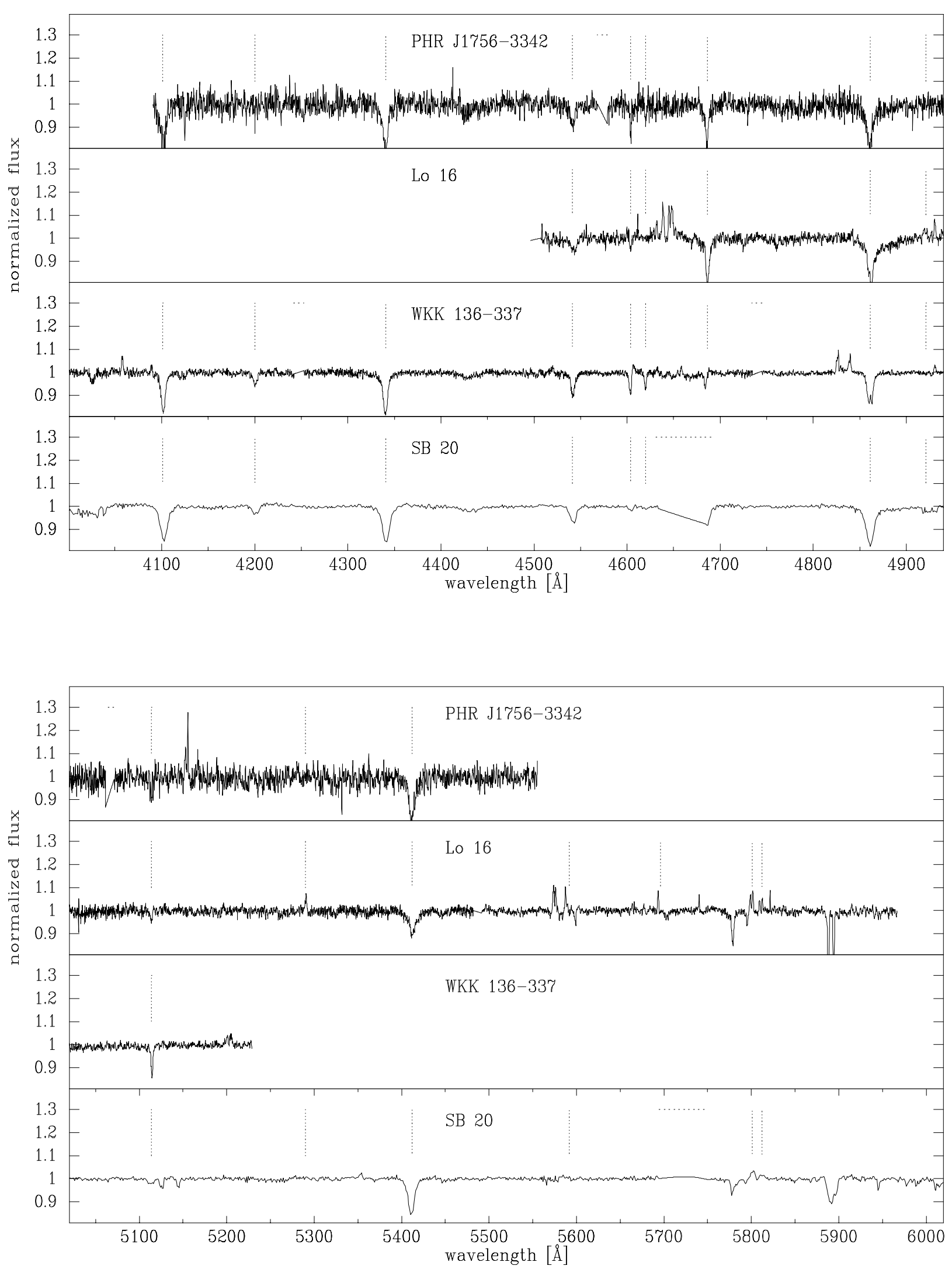

Fig. A.3. Same as Fig. A.1. 
A\&A 614, A135 (2018)
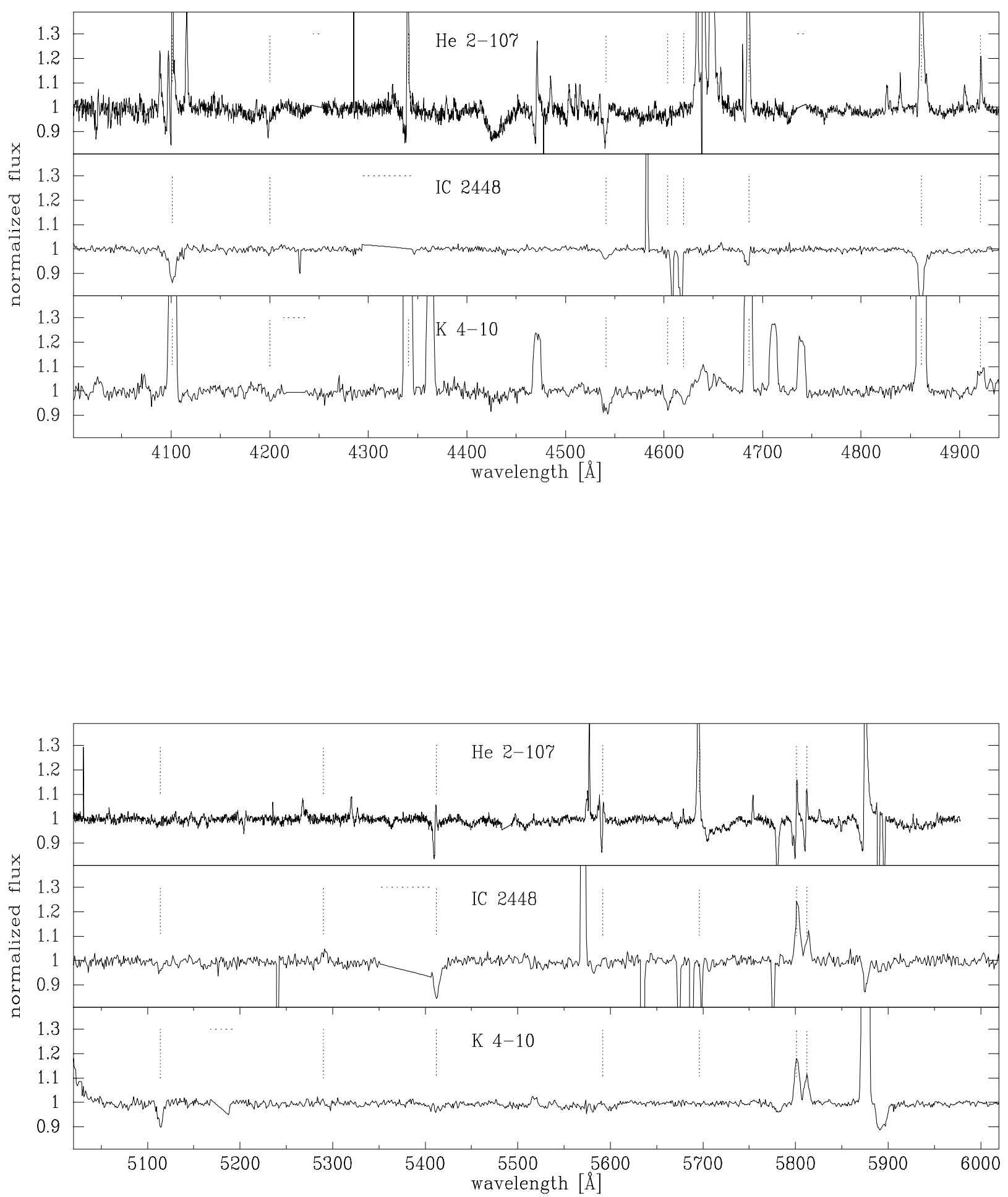

Fig. A.4. Same as Fig. A.1. 
W. Weidmann et al.: Towards an improvement in the spectral description of central stars of planetary nebulae
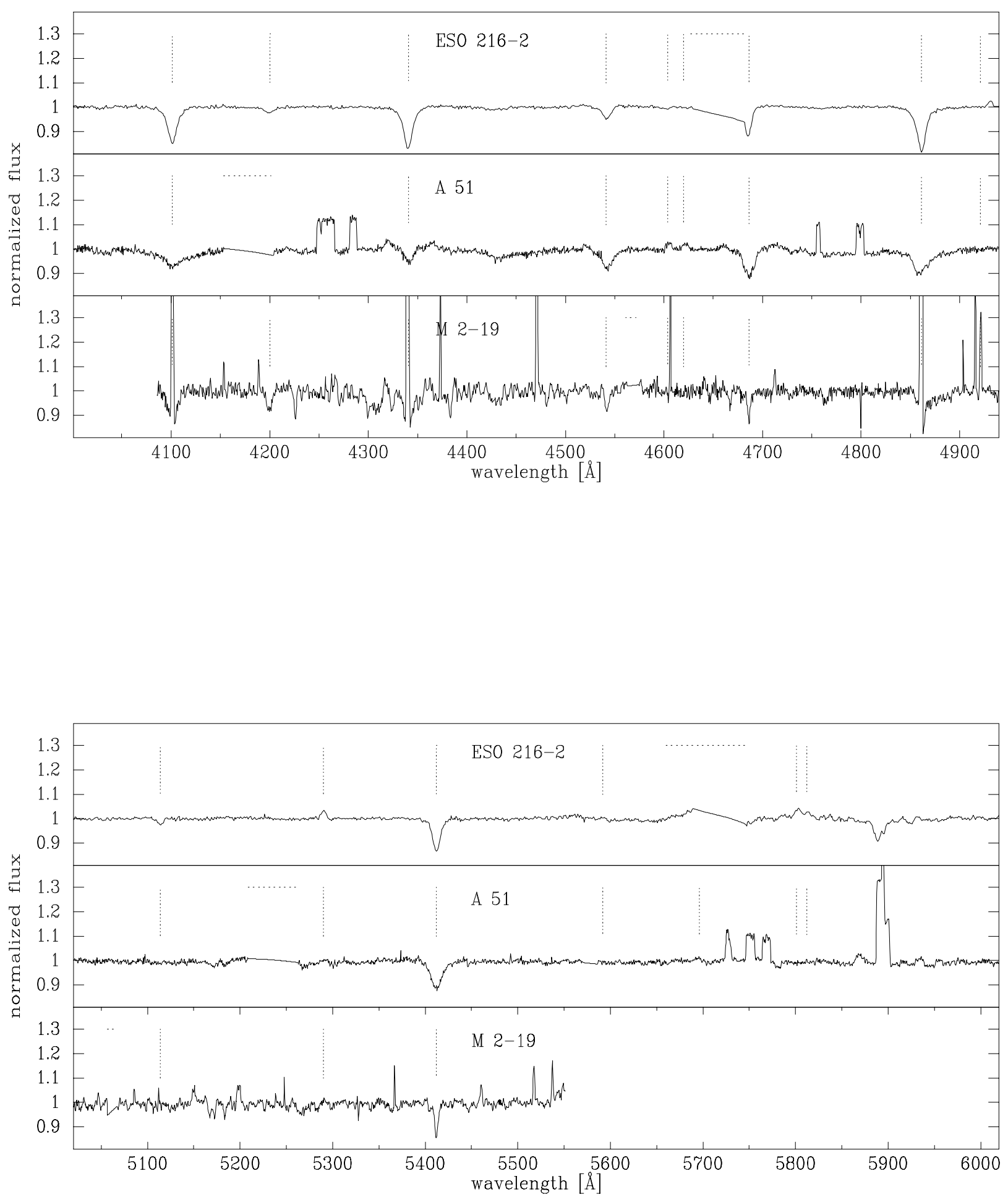

Fig. A.5. Same as Fig. A.1. 
A\&A 614, A135 (2018)
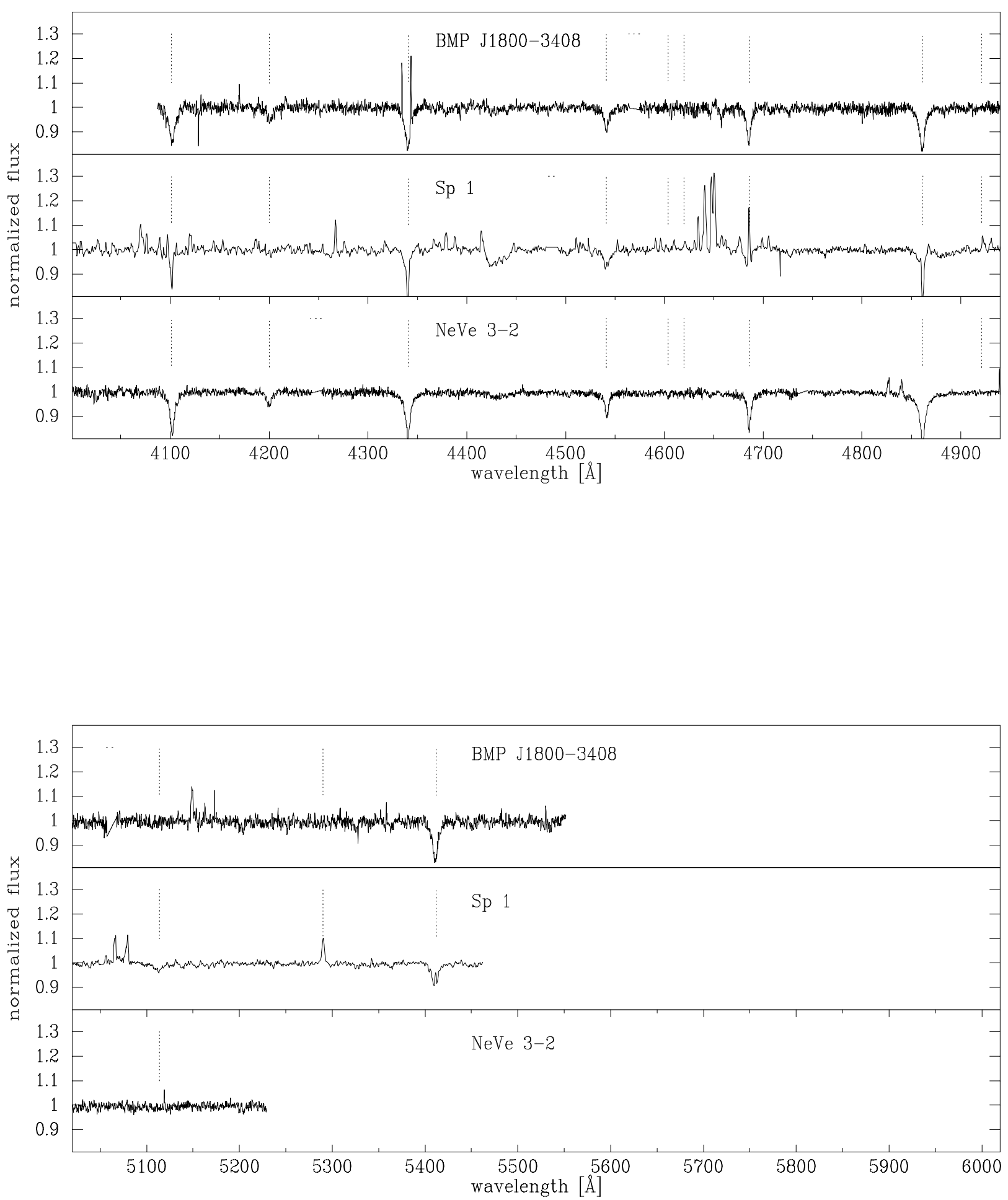

Fig. A.6. Same as Fig. A.1. 
W. Weidmann et al.: Towards an improvement in the spectral description of central stars of planetary nebulae
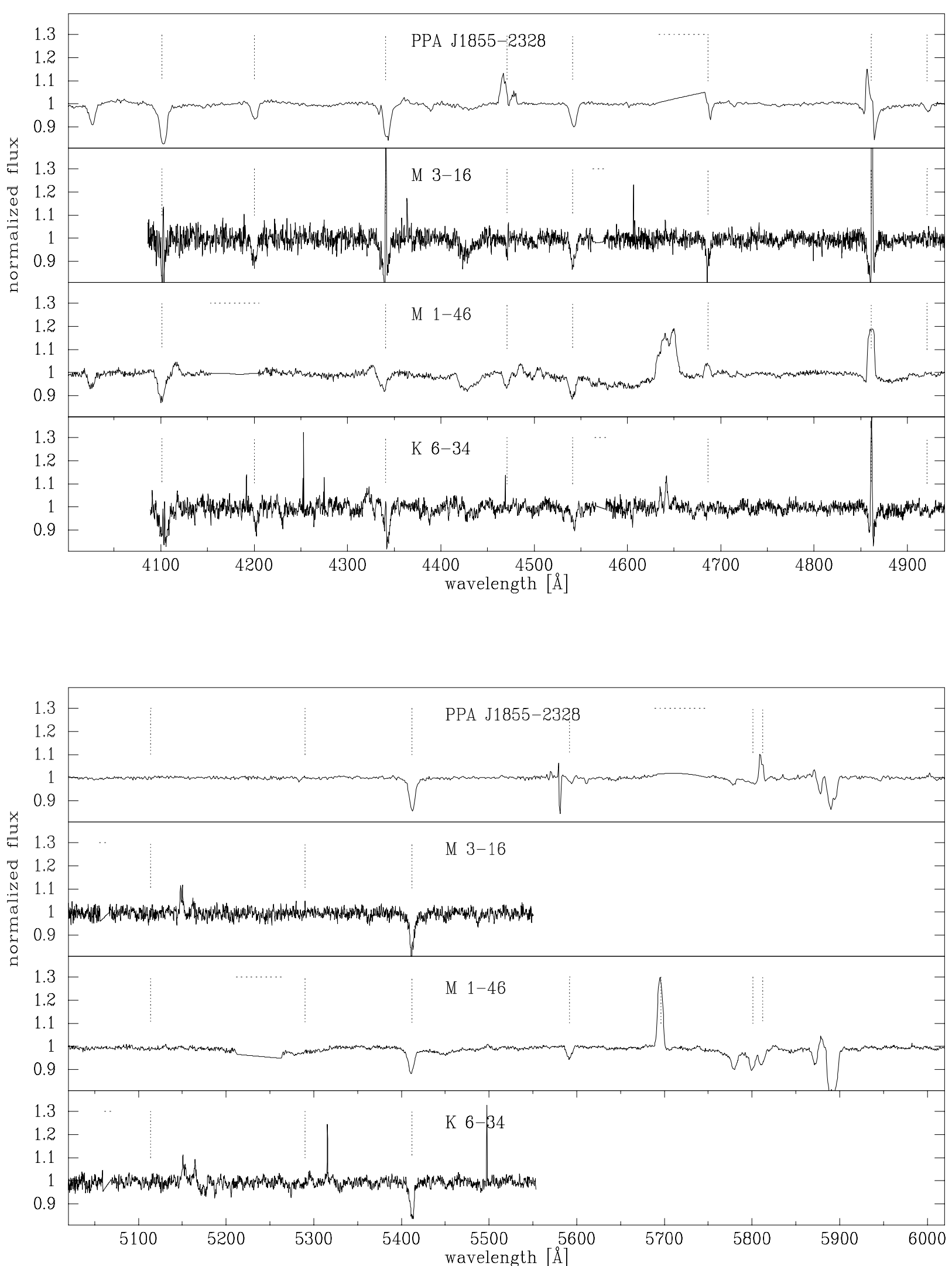

Fig. A.7. Normalized spectra of intermediate O-type CSPN (see Table A.3). The interstellar absorption bands at $\lambda 4428$, the complex at 5780 and 5890-6 are not indicated. The most important spectral features (absorption and emission) identified are: $\mathrm{H} \beta, \mathrm{H} \gamma, \mathrm{H} \delta, \mathrm{He}$ I $\lambda 4471$ and $\lambda 4921$, He II $\lambda 4200,4542$, and 4686, for the blue spectral range (top panel), and Ov $\lambda 5114$, O vi $\lambda 5290, \mathrm{He}_{\text {II }} \lambda 5412, \mathrm{O}_{\text {III }} \lambda 5592, \mathrm{C}_{\text {III }} \lambda 5696$, and $\mathrm{C}_{\text {IV }}$ 15801-12, for the red spectral range (bottom panel). 
A\&A 614, A135 (2018)
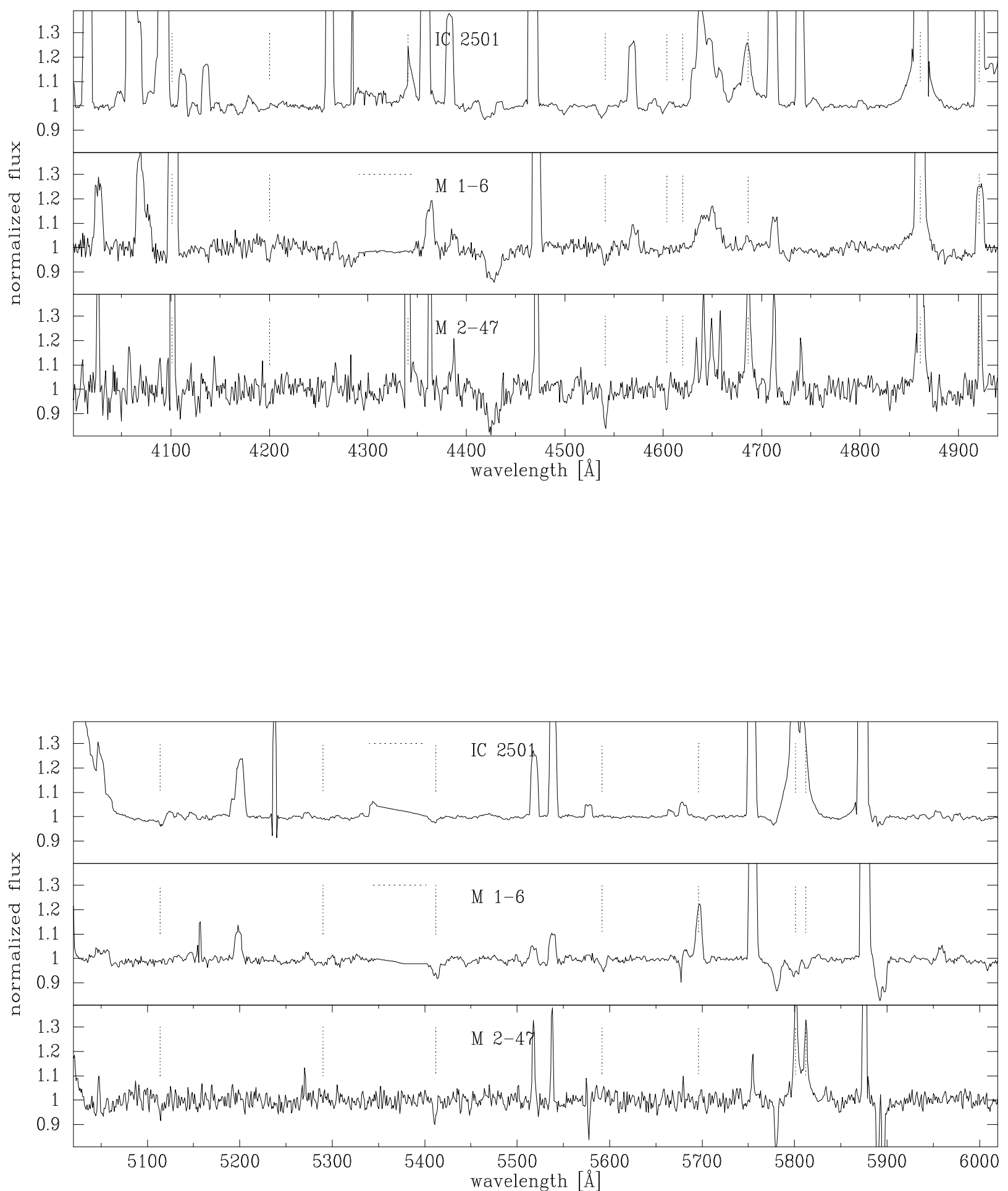

Fig. A.8. Normalized spectra of O-type CSPN (see Table A.3). In these objects we could not detect the Balmer series. The interstellar absorption bands at $\lambda 4428$, the complex at 5780 and 5890-6 are not indicated. The most important spectral features (absorption and emission) identified are: He II $\lambda 4200,4542$, and 4686, Nv $\lambda 4604-19$, for the blue spectral range (top panel), and O v $\lambda 5114$, O vi $\lambda 5290, \mathrm{He}_{\text {II }} \lambda 5412, \mathrm{O}_{\text {III }} \lambda 5592, \mathrm{C}_{\text {III }}$ 15696, C Iv $\lambda 5801-12$, for the red spectral range (bottom panel). 
W. Weidmann et al.: Towards an improvement in the spectral description of central stars of planetary nebulae
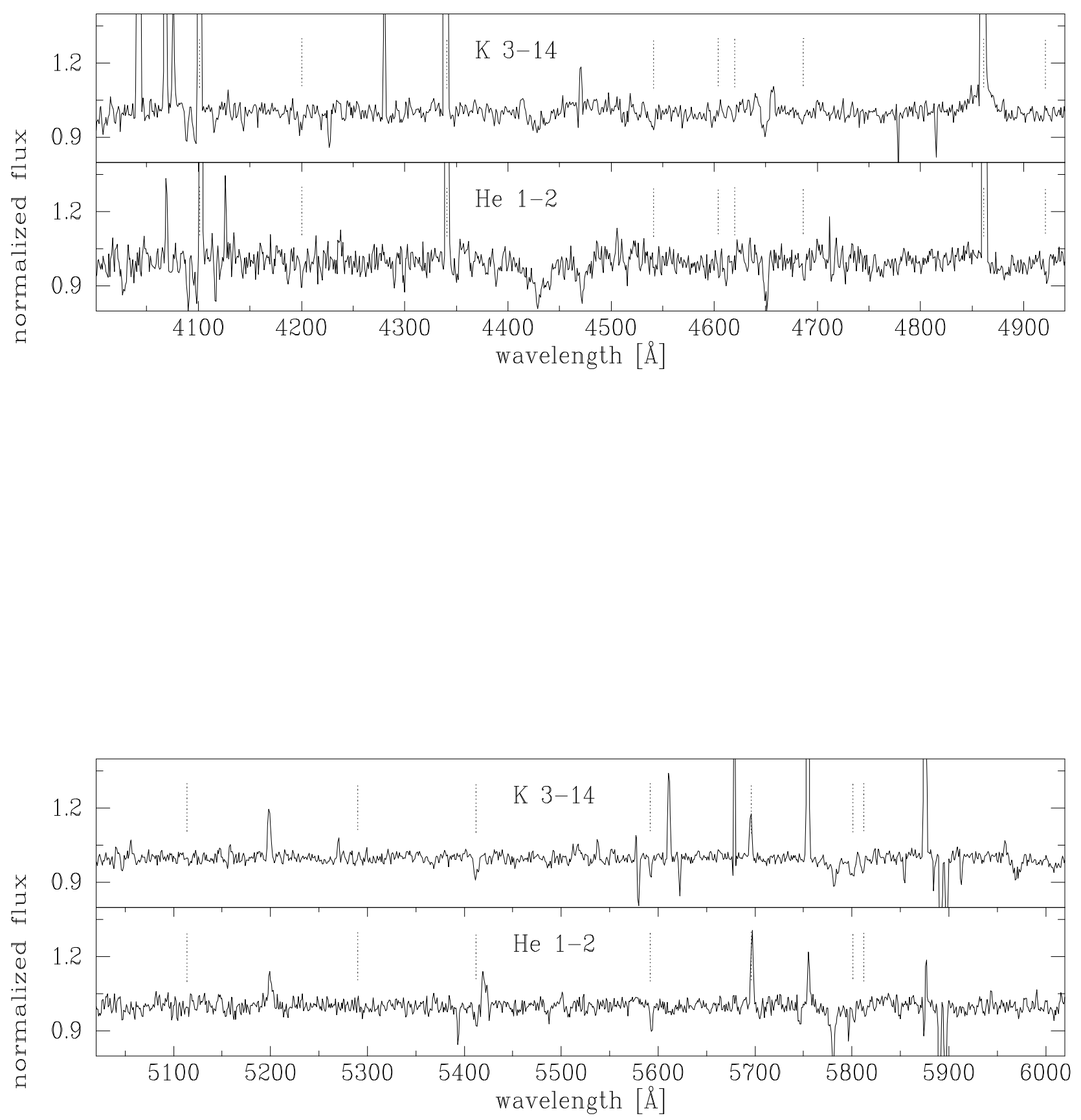

Fig. A.9. Normalized spectra of O-type CSPN (see Table A.3), in these objects we could not detect the Balmer serie. The interstellar absorption bands at $\lambda 4428$, the complex at 5780 and 5890-6 are not indicated. The most important spectral features (absorption and emission) identified are: He II $\lambda 4200,4542$, and 4686, N v $\lambda 4604-19$ for the blue spectral range (top panel), and same features as Fig. A.1 for the red spectral range (bottom panel). 
A\&A 614, A135 (2018)
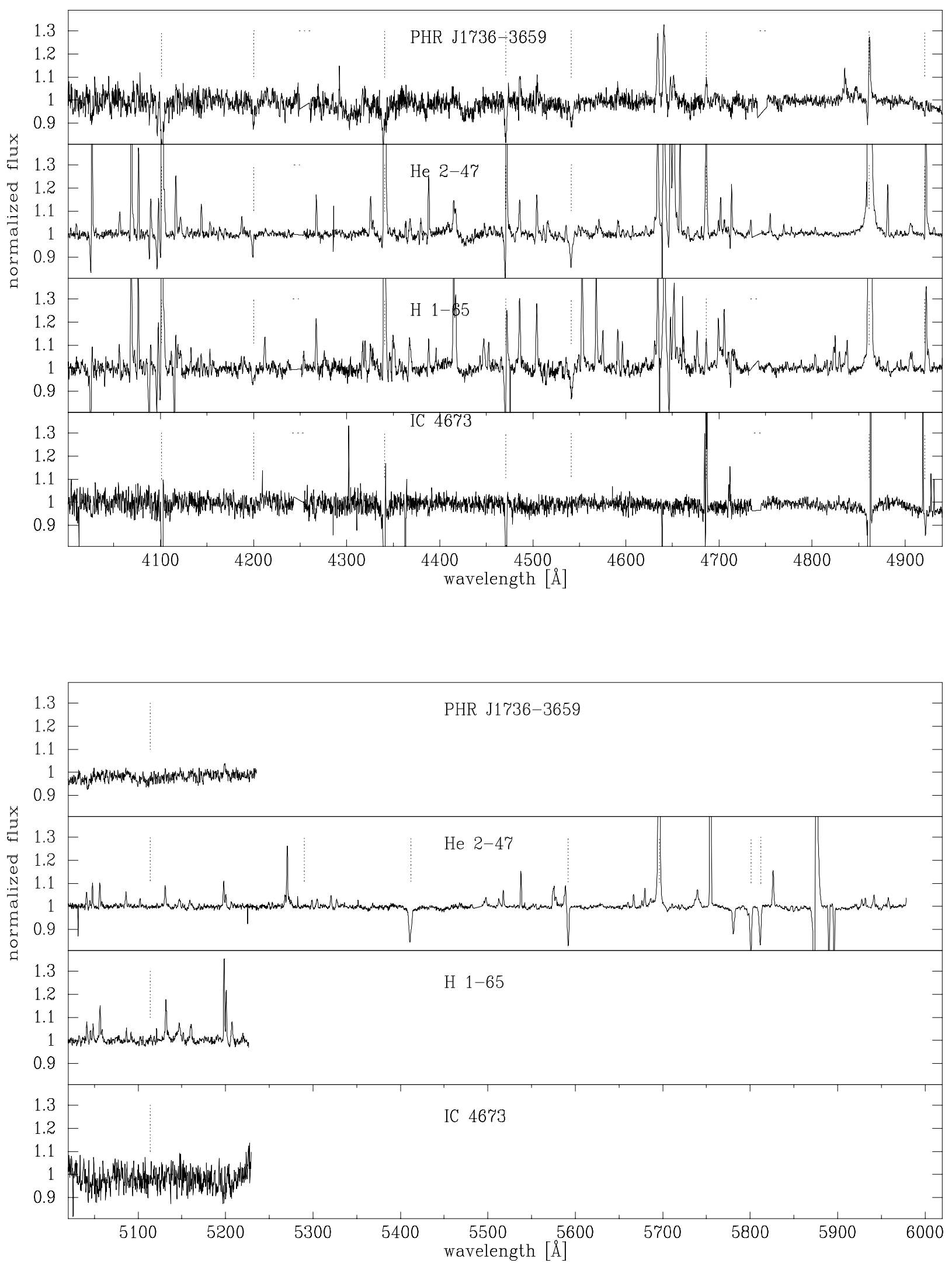

Fig. A.10. Normalized spectra of late O-type CSPN (see Table A.3). The interstellar absorption bands at $\lambda 4428$, the complex at 5780 and 5890-6 are not indicated. The most important spectral features (absorption and emission) identified are: $\mathrm{H} \beta, \mathrm{H} \gamma, \mathrm{H} \delta, \mathrm{He}$ I $\lambda 4471$, and $\lambda 4921, \mathrm{He}$ II $\lambda 4200$, 4542, and 4686, for the blue spectral range (top panel), and the same spectral features as Fig. A.1 for the red spectral range (bottom panel). 
W. Weidmann et al.: Towards an improvement in the spectral description of central stars of planetary nebulae
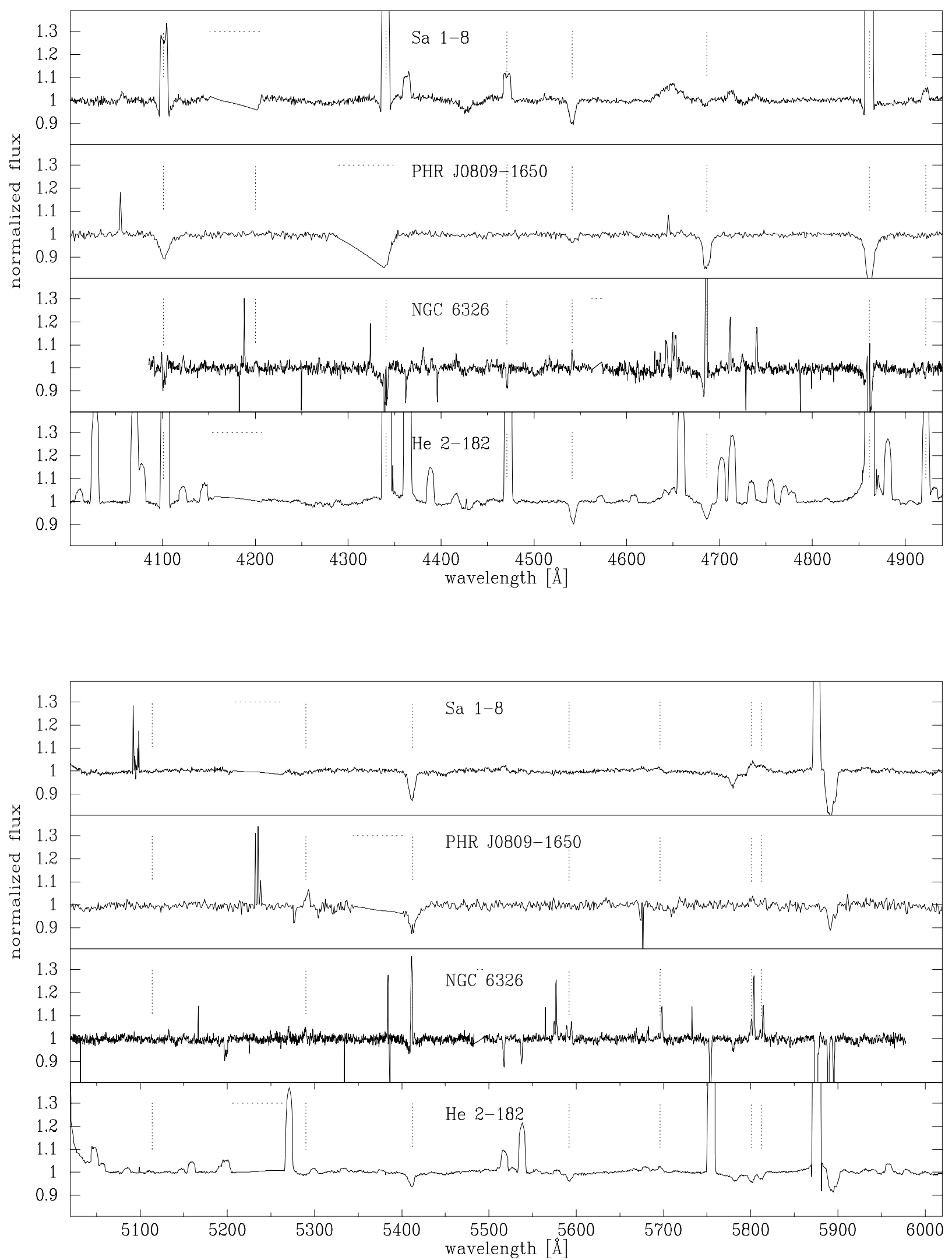

Fig. A.11. Normalized spectra of intermediate/late O-type CSPN (see Table A.3). Spectral features for blue and red spectral regions as Fig. A.10. 
A\&A 614, A135 (2018)
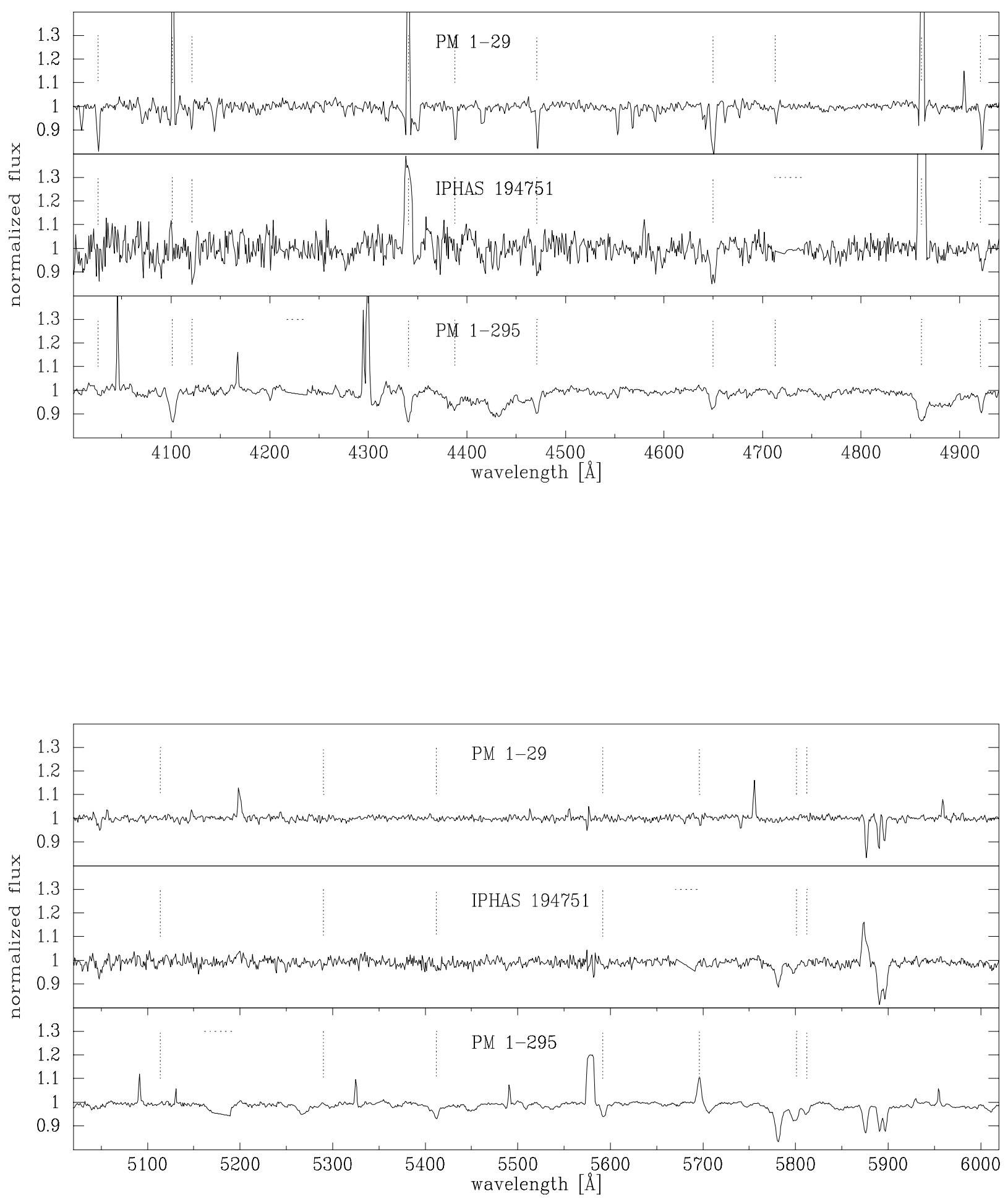

Fig. A.12. Normalized spectra of early B-type CSPN (see Table A.3). The interstellar absorption bands at $\lambda 4428$, the complex at 5780 and 5890-6 are not indicated. The most important spectral features (absorption and emission) identified are: $\mathrm{H} \beta, \mathrm{H} \gamma, \mathrm{H} \delta, \mathrm{He}$ I $\lambda 4026,4121,4387,4471,4713$, and 4921, C III $\lambda$ 4650, for the blue spectral range (top panel), and same features as Fig. A.1 for the red spectral range (bottom panel). 
W. Weidmann et al.: Towards an improvement in the spectral description of central stars of planetary nebulae
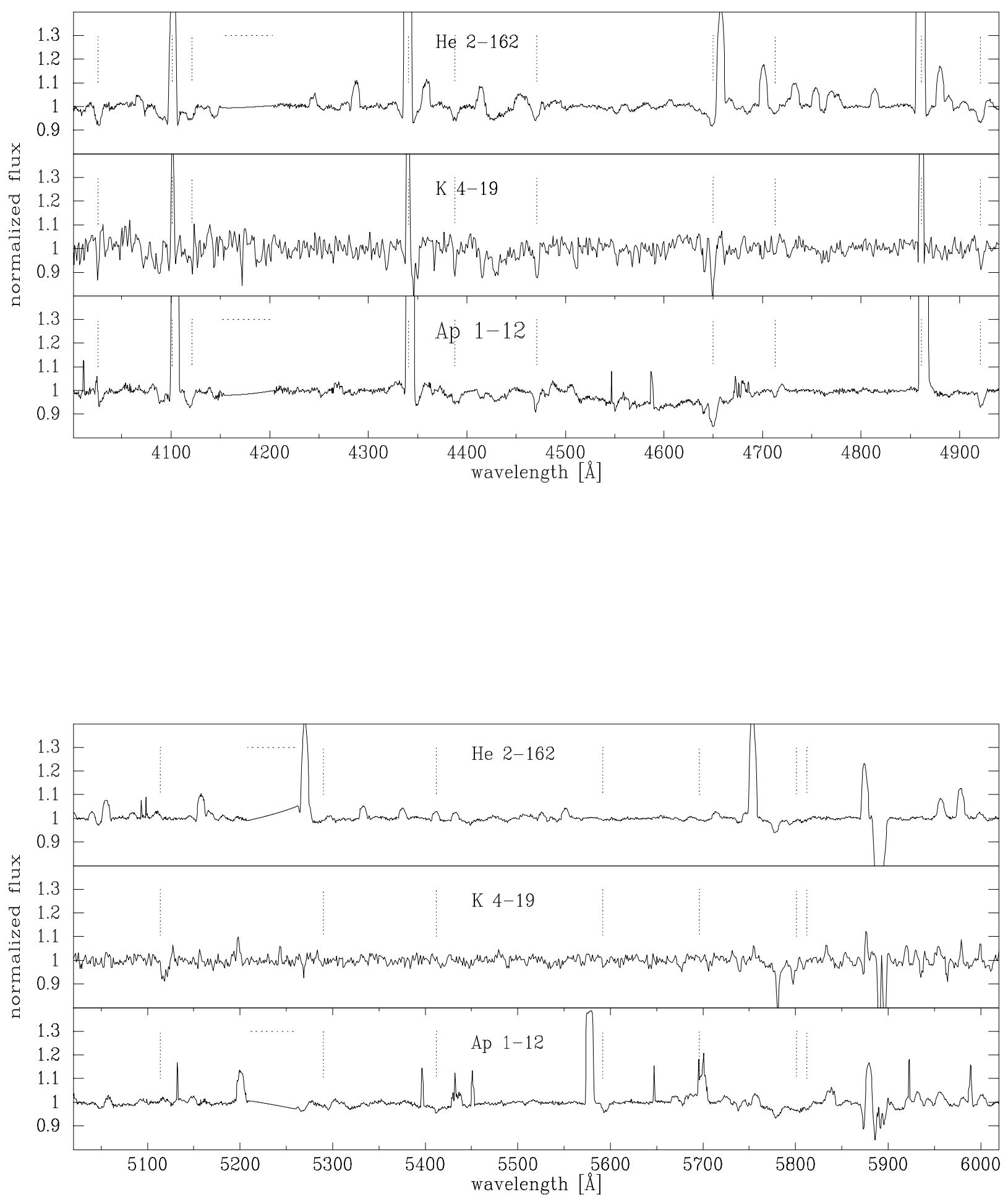

Fig. A.13. Same as Fig. A.12. 
A\&A 614, A135 (2018)
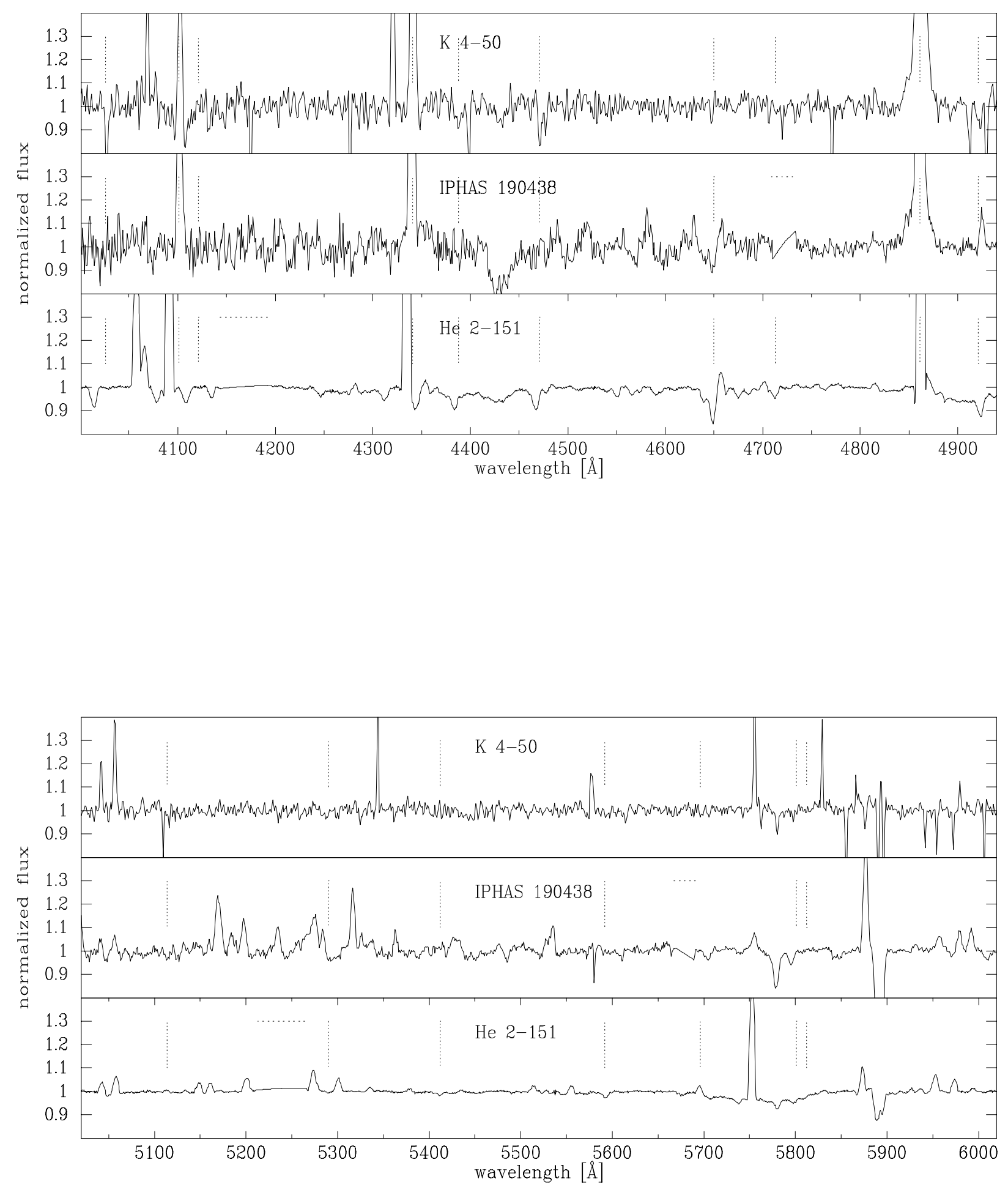

Fig. A.14. Same as Fig. A.12. 
W. Weidmann et al.: Towards an improvement in the spectral description of central stars of planetary nebulae
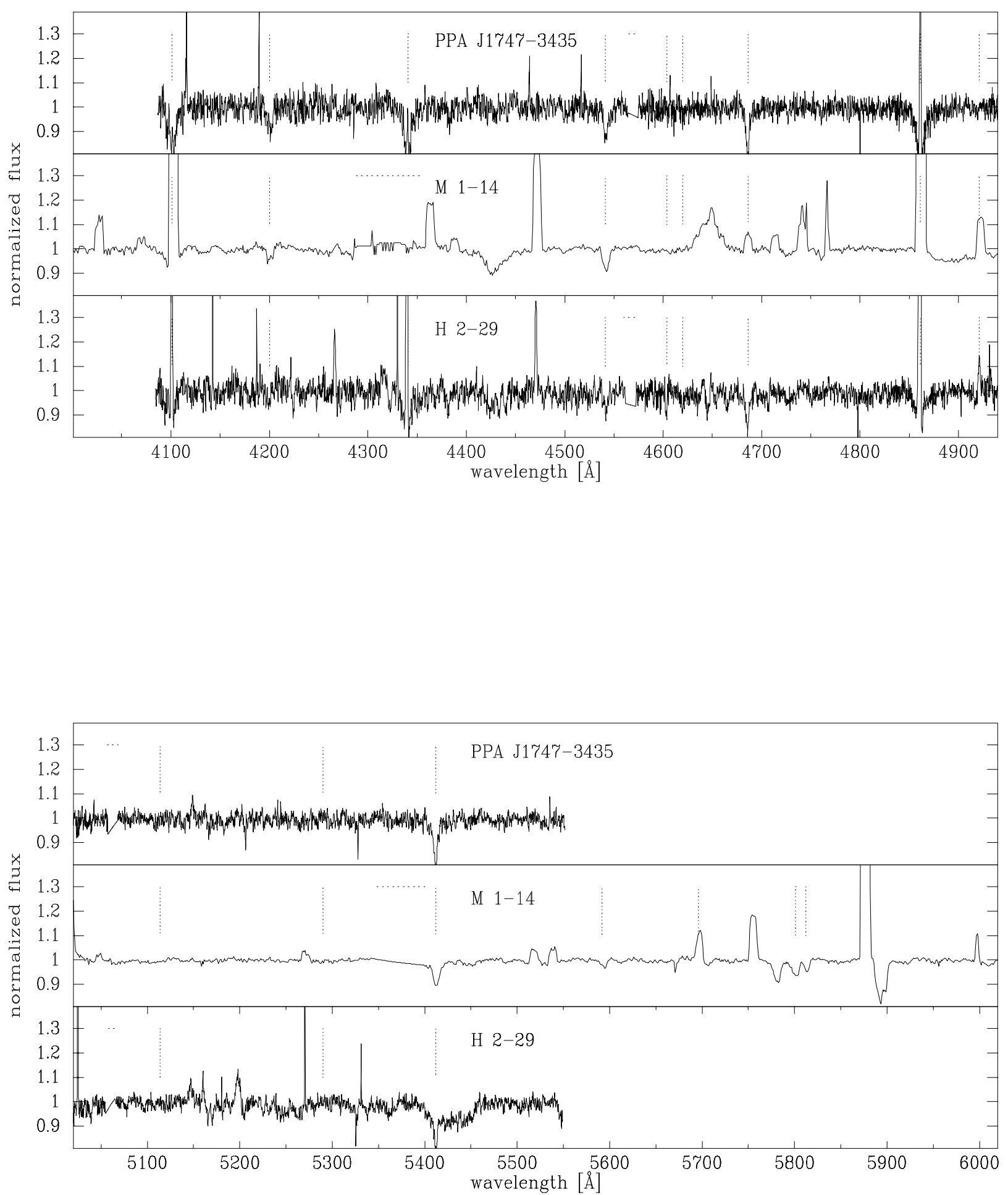

Fig. A.15. Normalized spectra of O(H)-type CSPN (see Table A.3). In these objects was not possible identify the sub-type of the O-type star. Blue and red spectral ranges features as in Fig. A.1. 
A\&A 614, A135 (2018)
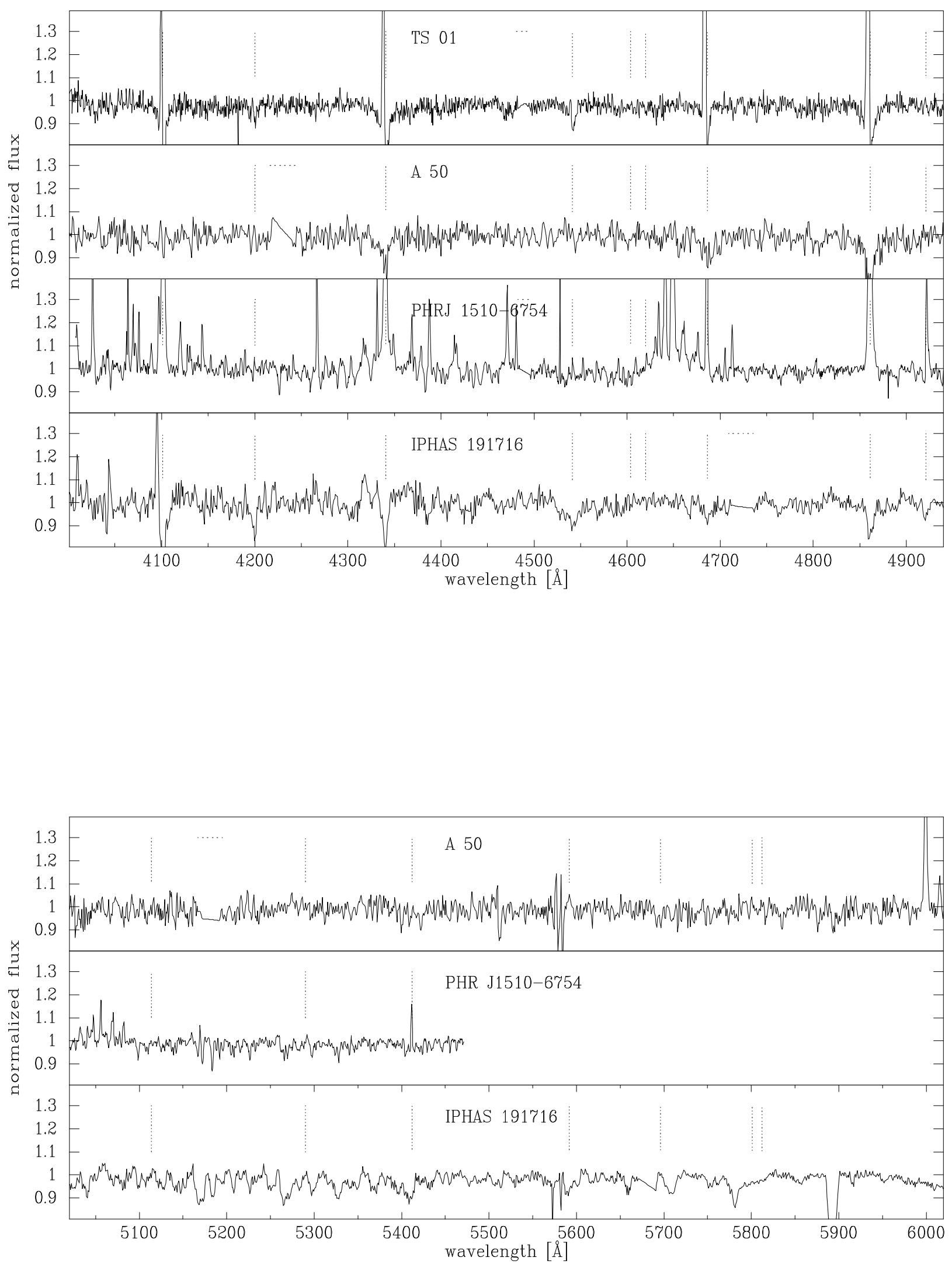

Fig. A.16. Same as Fig. A.15. 
W. Weidmann et al.: Towards an improvement in the spectral description of central stars of planetary nebulae
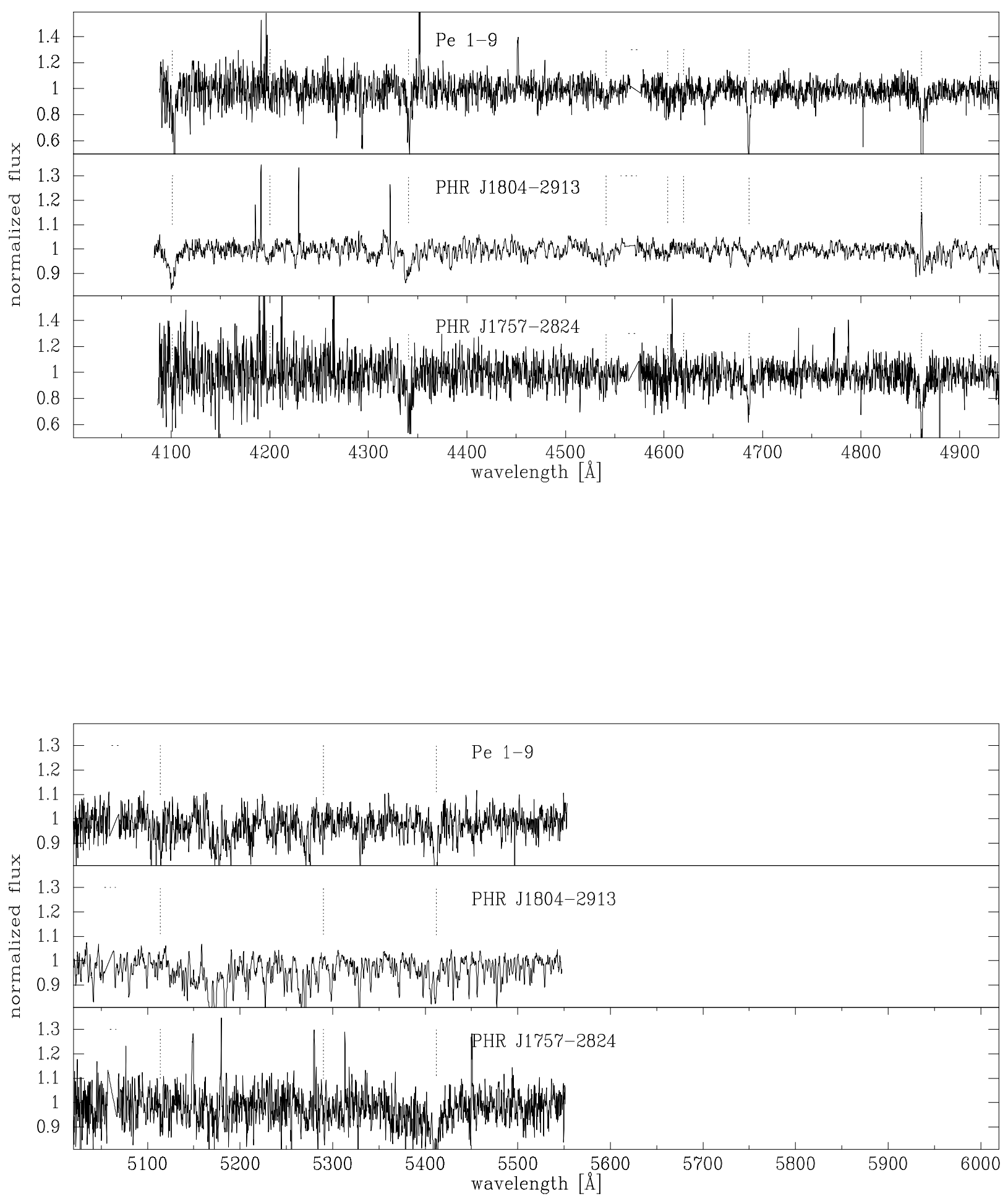

Fig. A.17. Same as Fig. A.15. 
A\&A 614, A135 (2018)
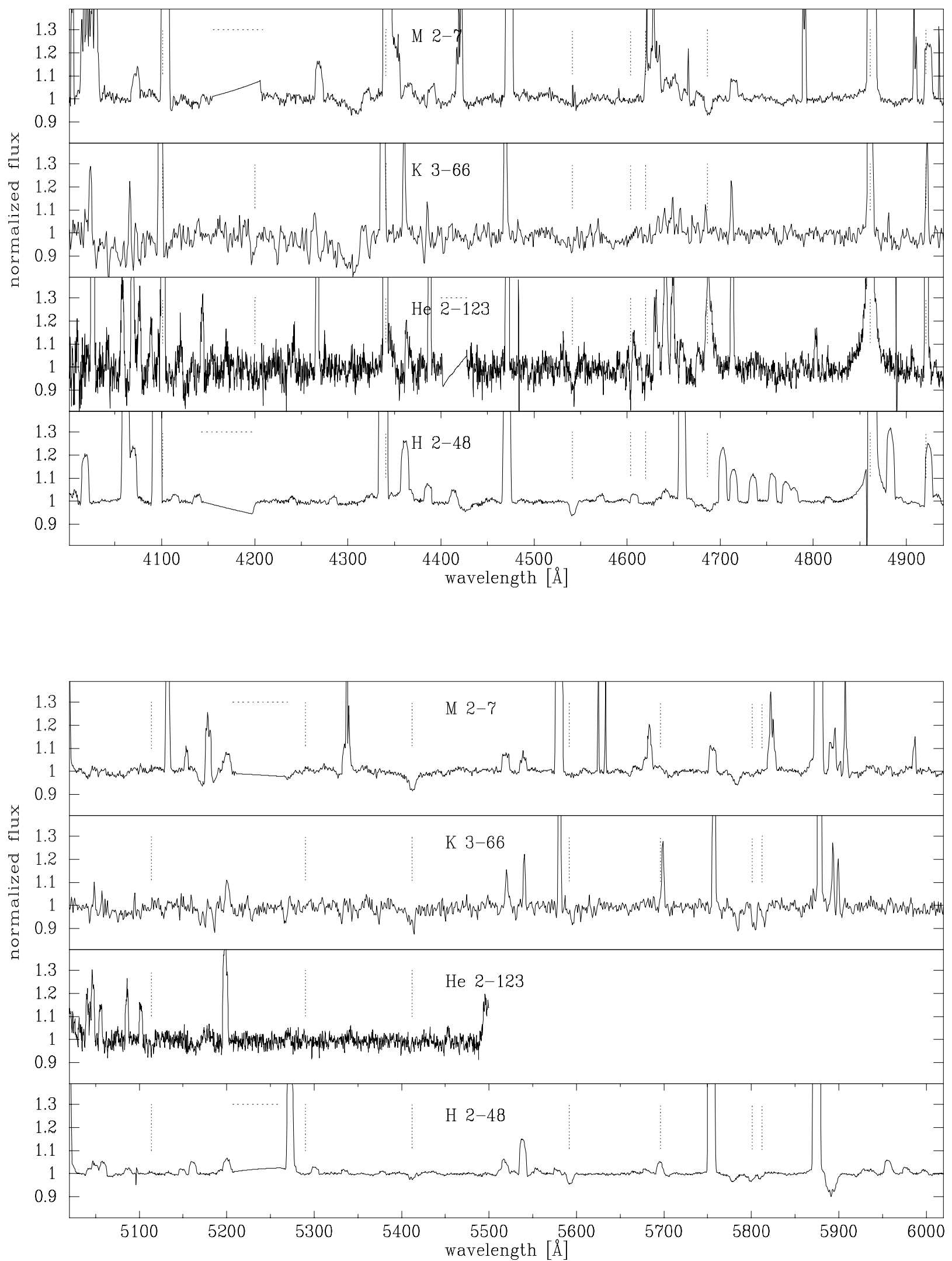

Fig. A.18. Normalized spectra of O-type CSPN (see Table A.3). For these objects we only detect He II absorption lines. Blue and red spectral ranges features as Fig. A.1. 
W. Weidmann et al.: Towards an improvement in the spectral description of central stars of planetary nebulae
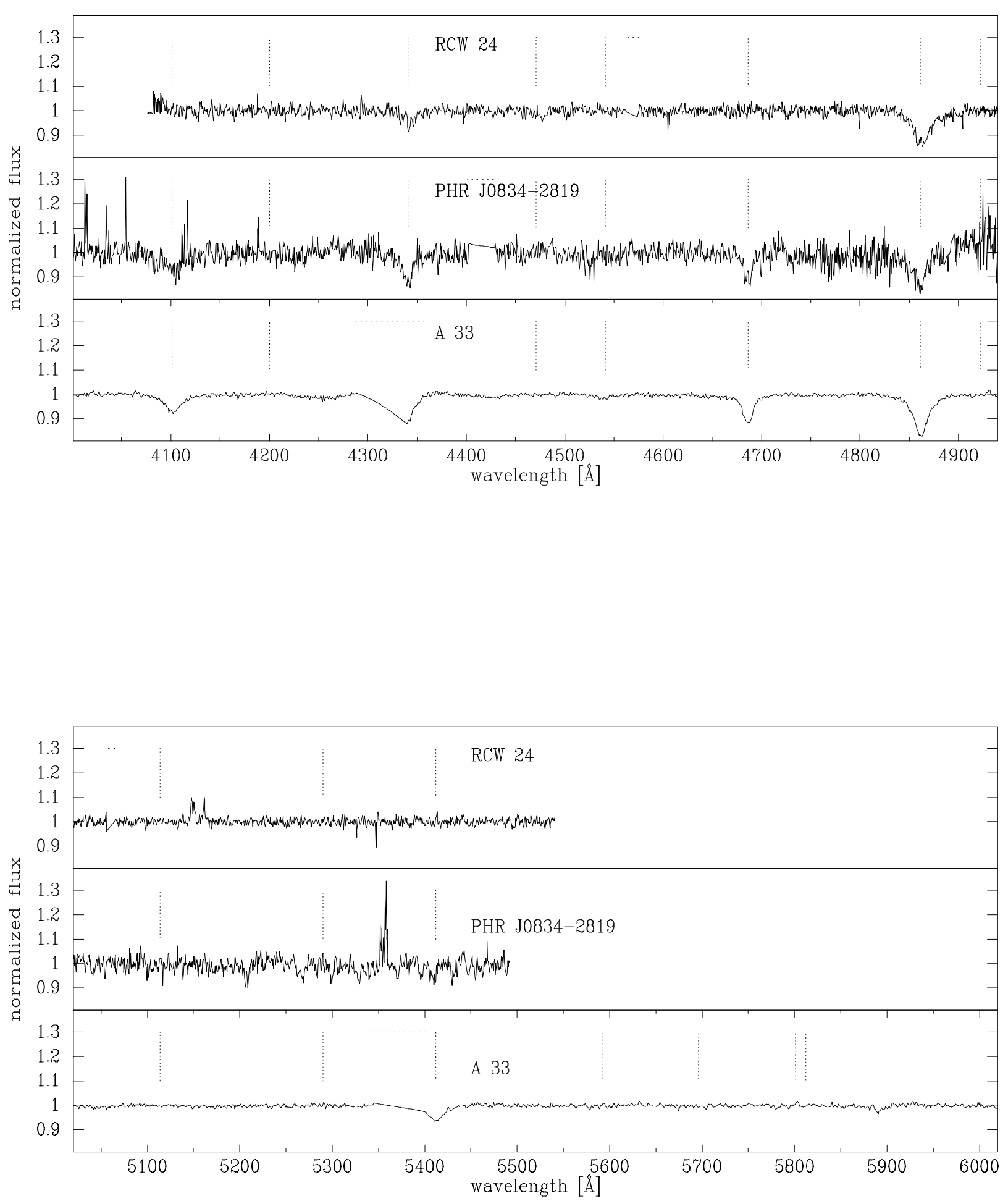

Fig. A.19. Normalized spectra of WD CSPN (see Table A.3). You should note the wide absorption lines. The interstellar absorption bands at $\lambda 4428$, the complex at 5780 and 5890-6 are not indicated. The most important spectral features (absorption and emission) identified are $\mathrm{H} \beta$, $\mathrm{H} \gamma$, $\mathrm{H} \delta$, Не $\mathrm{I} \lambda 4471$, and $\lambda 4921, \mathrm{He}$ I $\lambda 4200,4542$, and 4686, for the blue spectral range. Red range as Fig. A.1. 
A\&A 614, A135 (2018)
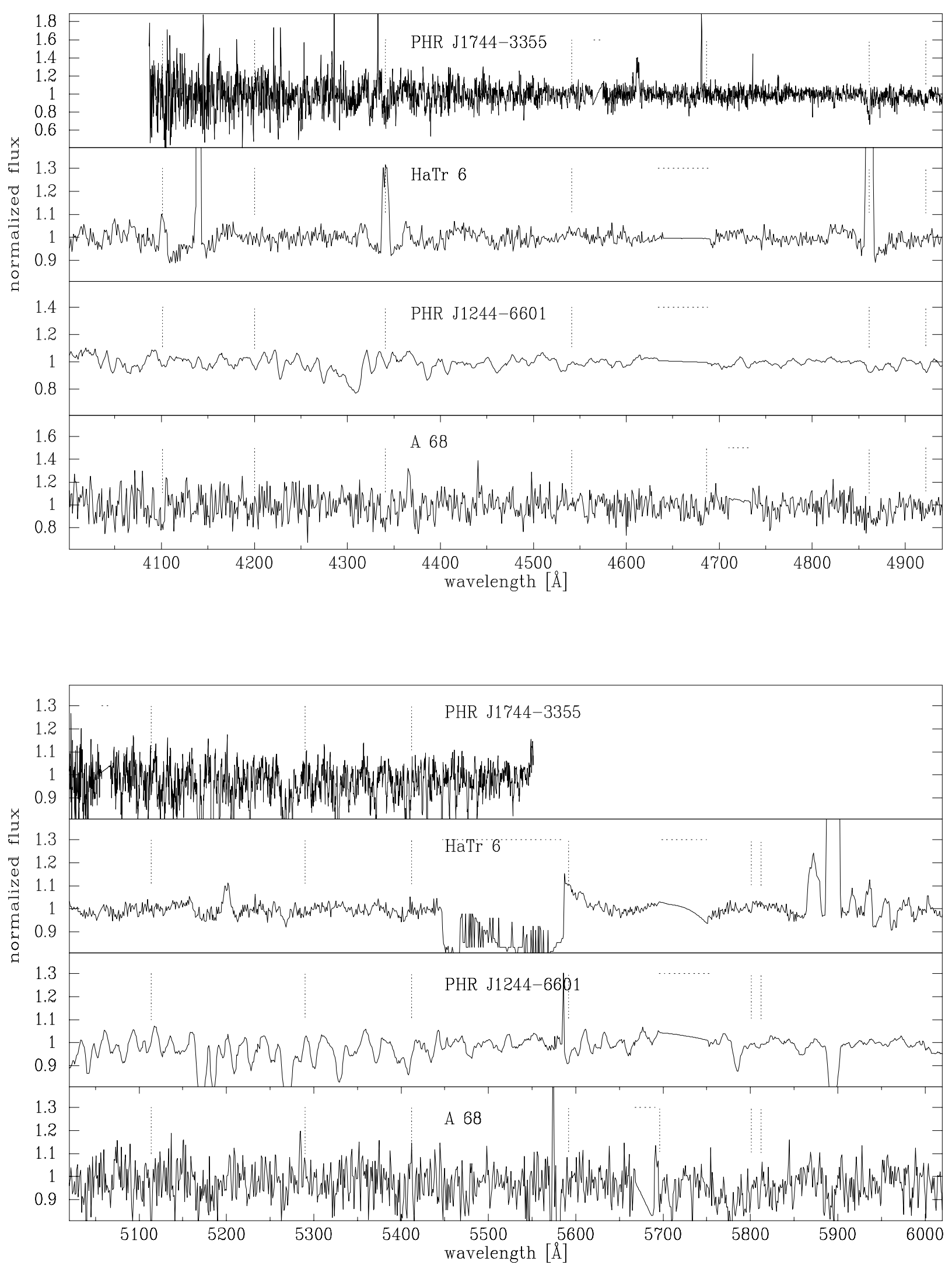

Fig. A.20. Normalized spectra of H-rich CSPN (see Table A.3). For these objects we only detect the Balmer series. The interstellar absorption bands at 24428 , the complex at 5780 and 5890-6 are not indicated. Blue and red spectral ranges features as Fig. A.1. 
W. Weidmann et al.: Towards an improvement in the spectral description of central stars of planetary nebulae
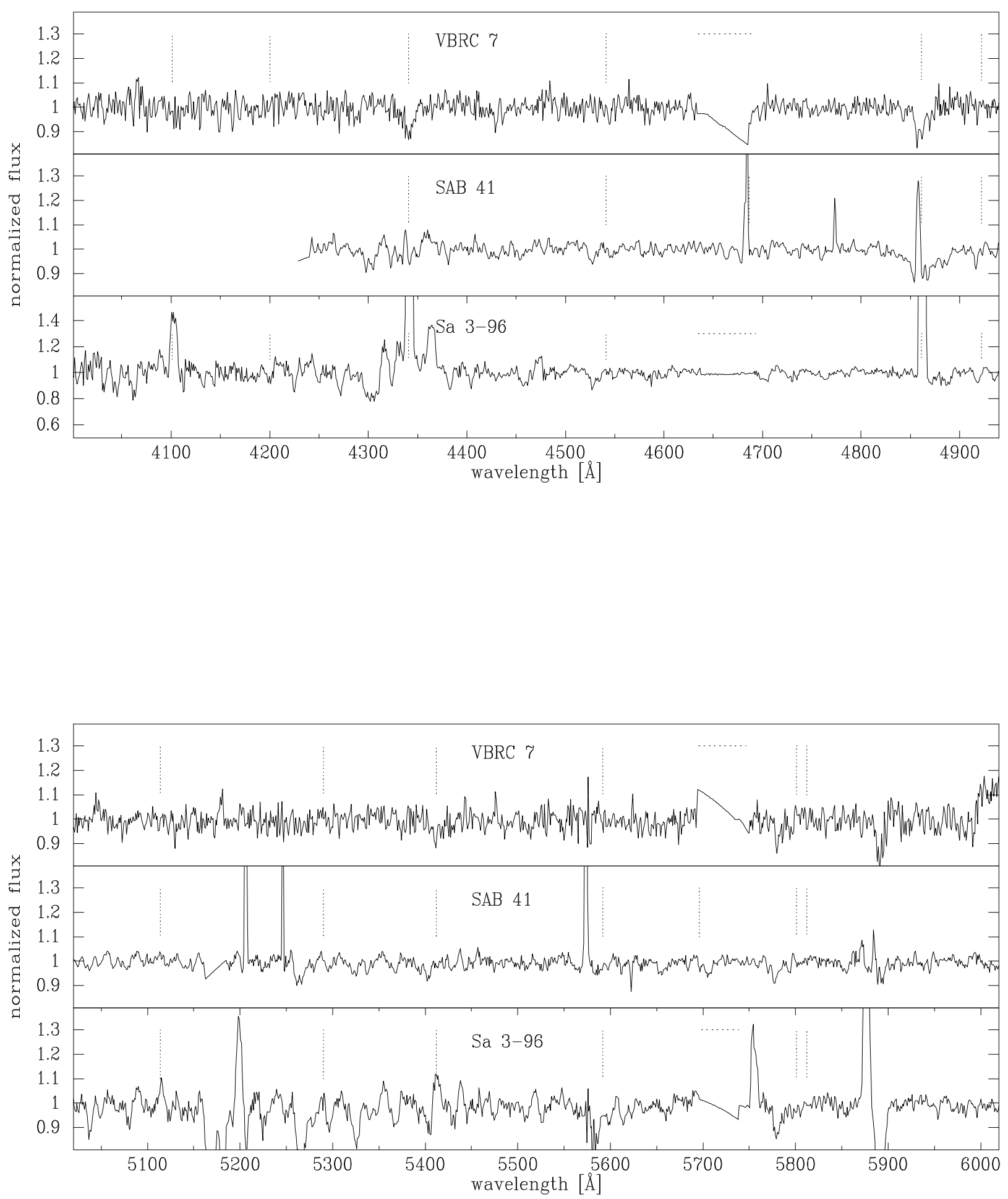

Fig. A.21. Same as Fig. A.20. 

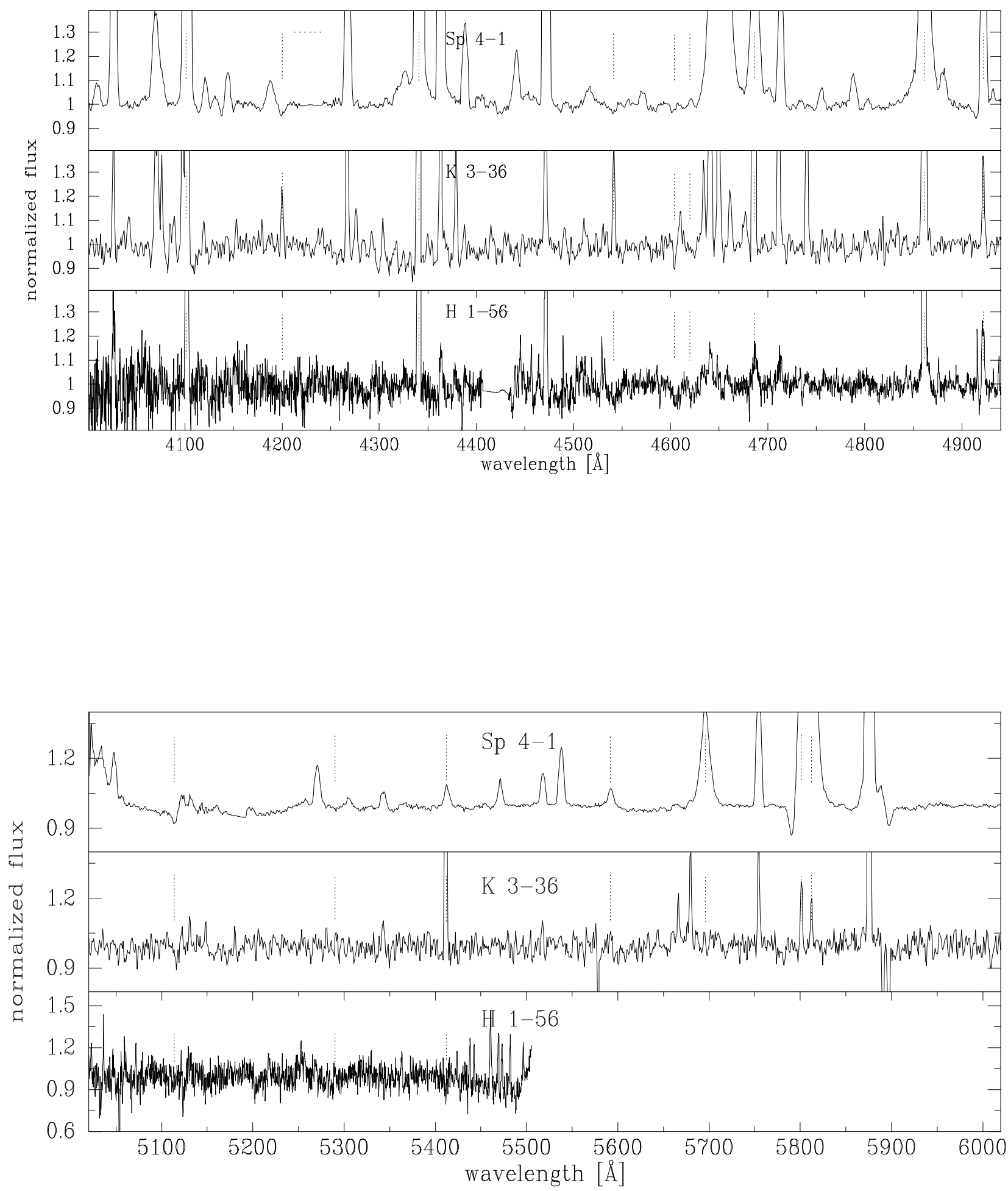

Fig. A.22. Normalized spectra of possible O-type CSPN (see Table A.3). Blue and red spectral ranges features as Fig. A.1. 
W. Weidmann et al.: Towards an improvement in the spectral description of central stars of planetary nebulae
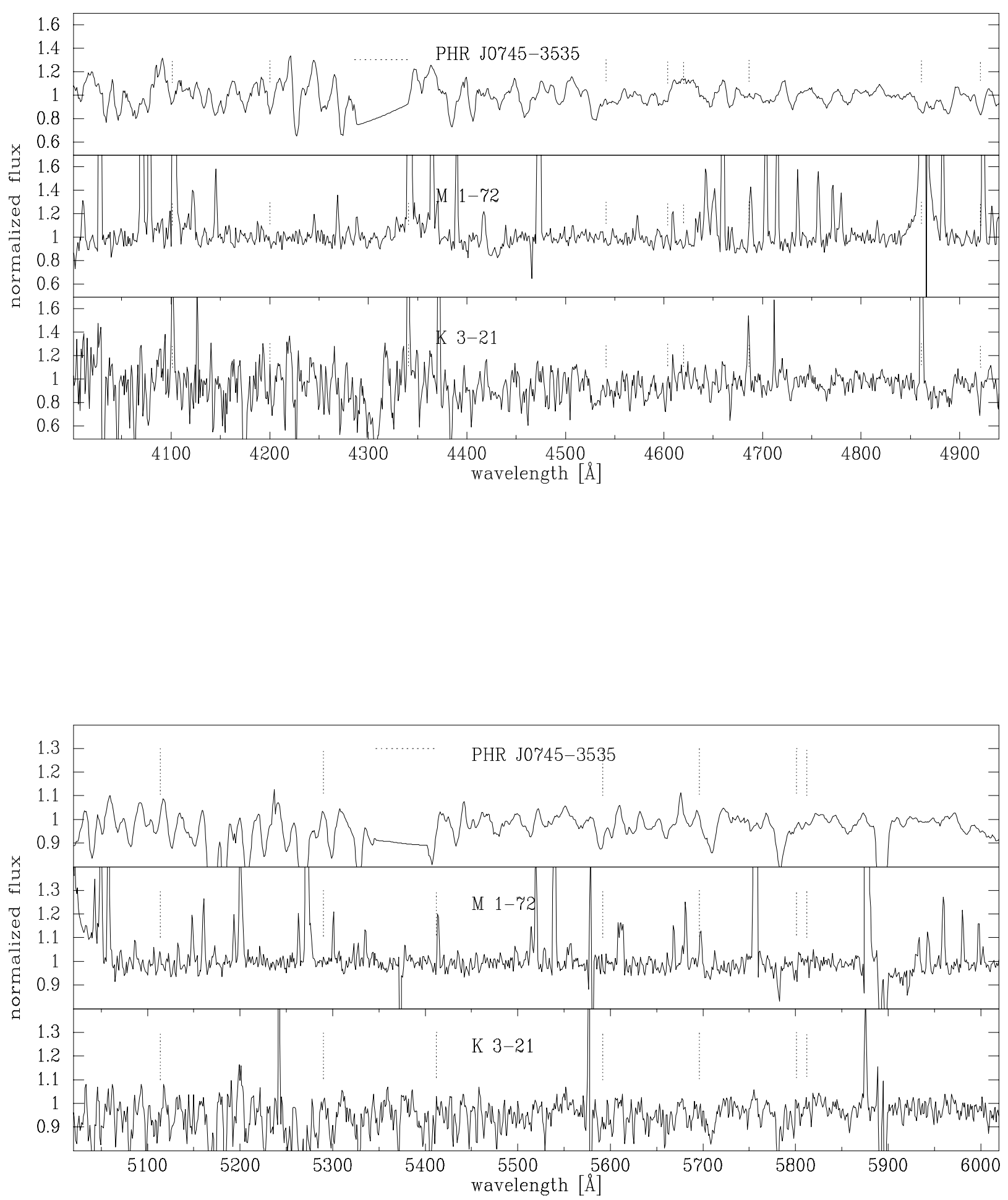

Fig. A.23. Normalized spectra of continuum CSPN (see Table A.3). Blue and red spectral regions features as Fig. A.1. 
A\&A 614, A135 (2018)
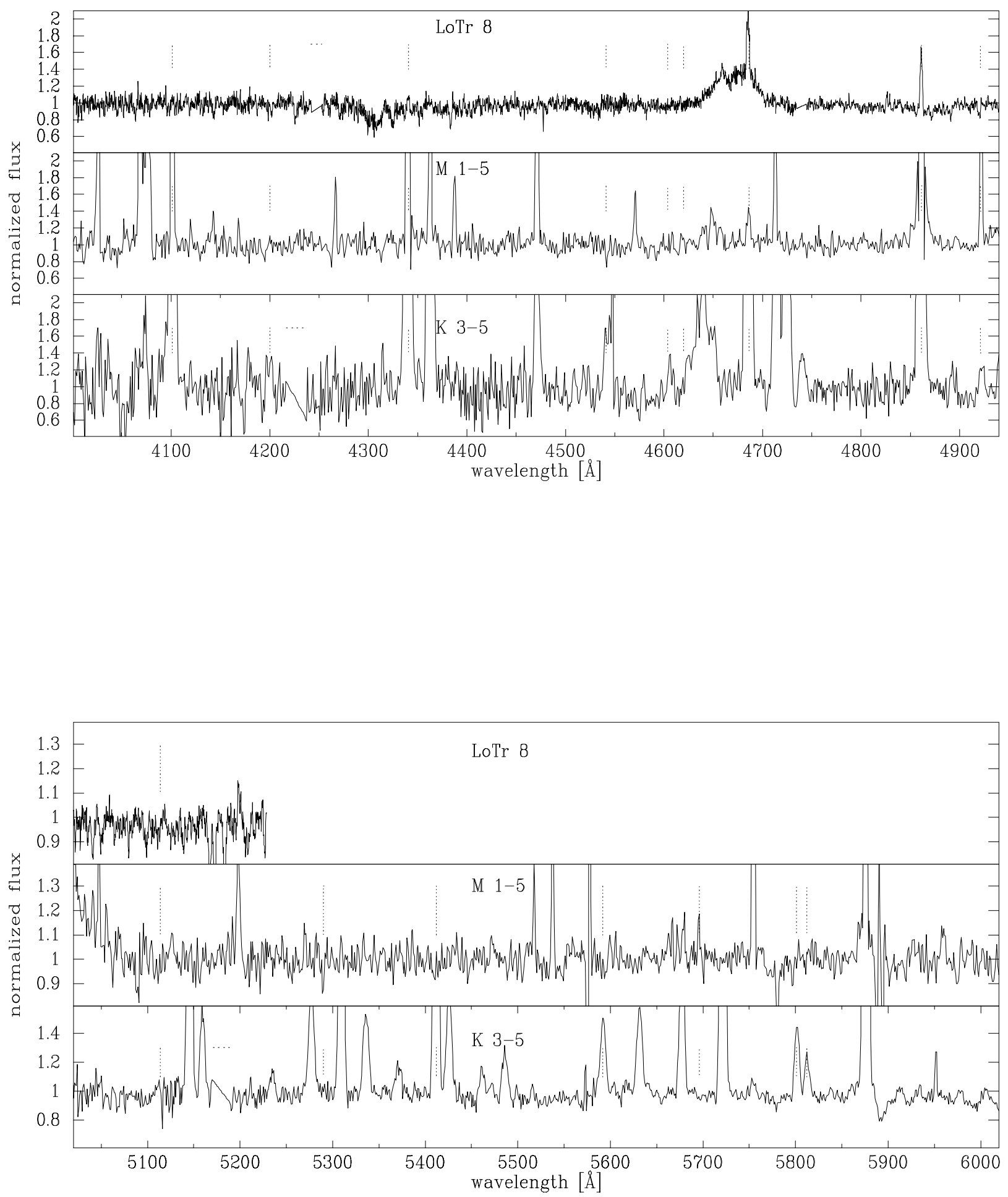

Fig. A.24. Normalized spectra of emission lines CSPN (see Table A.3). Blue and red spectral regions features as Fig. A.1. 